\title{
Invisible axions and large-radius compactifications
}

\author{
Keith R. Dienes* \\ Theory Division, CERN, CH-1211 Geneva 23, Switzerland \\ and Department of Physics, University of Arizona, Tucson, Arizona 85721 \\ Emilian Dudas ${ }^{\dagger}$ \\ LPT, Bât. 210, Université Paris-Sud, F-91405, Orsay Cedex, France \\ Tony Gherghetta \\ Theory Division, CERN, CH-1211 Geneva 23, Switzerland \\ and IPT, University of Lausanne, CH-1015 Lausanne, Switzerland \\ (Received 5 June 2000; published 23 October 2000)
}

\begin{abstract}
We study some of the novel effects that arise when the QCD axion is placed in the "bulk" of large extra spacetime dimensions. First, we find that the mass of the axion can become independent of the energy scale associated with the breaking of the Peccei-Quinn symmetry. This implies that the mass of the axion can be adjusted independently of its couplings to ordinary matter, a feature which is not possible in four dimensions and which may contribute to axion invisibility. Second, we discuss the new phenomenon of laboratory axion oscillations (analogous to neutrino oscillations), and show that these oscillations cause laboratory axions to “decohere"' extremely rapidly as a result of Kaluza-Klein mixing. This decoherence may also be a contributing factor to axion invisibility. Third, we discuss the role of Kaluza-Klein axions in axion-mediated processes and decays, and propose several experimental tests of the higher-dimensional nature of the axion. Finally, we show that under certain circumstances the presence of an infinite tower of Kaluza-Klein axion modes can significantly accelerate the dissipation of the energy associated with cosmological relic axion oscillations, thereby enabling the Peccei-Quinn symmetry-breaking scale to exceed the usual four-dimensional relic oscillation bounds. Together, these ideas therefore provide new ways of obtaining an "invisible" axion within the context of higher-dimensional theories with large-radius compactifications.
\end{abstract}

PACS number(s): 11.10.Kk, 11.25.Mj, 14.80.Mz

\section{INTRODUCTION}

One of the pressing theoretical issues that confronts the standard model of particle physics is to understand the origins of $C P$ violation. While the weak interaction is well understood to lead to $C P$ violation through complex phases in the Cabibbo-Kobayashi-Maskawa (CKM) fermion mass matrix, $C P$ violation may also independently arise from the physics of the strong interaction. Ordinarily, one might have assumed the strong interaction to conserve $C P$. However, the $U(1)$ problem [1] [namely, the inability to interpret the $\eta$ particle as the Nambu-Goldstone boson of a spontaneously broken $U(1)$ flavor symmetry] turns out to require a nontrivial QCD vacuum structure [2] which in turn naturally leads to $C P$ violation. Specifically, one finds that the effective Lagrangian describing the strong interaction should be augmented by an additional $C P$-violating contribution

$$
\mathcal{L}^{\mathrm{eff}}=\mathcal{L}_{\mathrm{QCD}}+\bar{\Theta} \frac{g^{2}}{32 \pi^{2}} F_{a}^{\mu \nu} \widetilde{F}_{\mu \nu a}
$$

where the $\bar{\Theta}$ parameter receives two contributions:

\footnotetext{
*Email address: dienes@ physics.arizona.edu

†Email address: emilian.dudas@th.u-psud.fr

†Email address: tony.gherghetta@cern.ch
}

$$
\bar{\Theta} \equiv \Theta+\operatorname{argdet} M \text {. }
$$

Here $\Theta$ is the strong-interaction $\Theta$-angle reflecting the nontrivial nature of the QCD vacuum, and $\operatorname{argdet} M$ (with $M$ denoting the CKM matrix) is the contribution arising from weak interactions. However, measurements of the neutron electric dipole moment place the stringent experimental bound

$$
\bar{\Theta} \lesssim 10^{-9} \text {. }
$$

Explaining the small size of $\bar{\Theta}$ is the strong $C P$ problem [3]. The most elegant explanation of the strong $C P$ problem is the Peccei-Quinn (PQ) mechanism [4], in which $\bar{\Theta}$ is set to zero dynamically as the result of a global, spontaneously broken $U(1)$ Peccei-Quinn symmetry. However, associated with this symmetry is a new Nambu-Goldstone boson, the axion $[5,6]$, which essentially replaces the $\bar{\Theta}$ parameter in the effective Lagrangian. This then results in an effective Lagrangian of the form [3]

$$
\mathcal{L}^{\mathrm{eff}}=\mathcal{L}_{\mathrm{QCD}}+\frac{1}{2} \partial_{\mu} a \partial^{\mu} a+\frac{a}{f_{\mathrm{PQ}}} \xi \frac{g^{2}}{32 \pi^{2}} F_{a}^{\mu \nu} \widetilde{F}_{\mu \nu a}
$$

where $f_{\mathrm{PQ}}$ is the axion decay constant, associated with the scale of PQ symmetry breaking. Here $\xi$ is a modeldependent parameter describing the PQ transformation prop- 
erties of the ordinary fermions, and we have not exhibited further Lagrangian terms which describe axion-fermion couplings. The mass of the axion is then expected to be of the order

$$
m_{a} \sim \frac{\Lambda_{\mathrm{QCD}}^{2}}{f_{\mathrm{PQ}}}
$$

where $\Lambda_{\mathrm{QCD}} \approx 250 \mathrm{MeV}$; likewise, the couplings of axions to fermions are suppressed by a factor of $1 / f_{\mathrm{PQ}}$. Thus, heavier scales for PQ symmetry breaking generally imply lighter axions which couple more weakly to ordinary matter.

Ordinarily, one might have liked to associate the scale $f_{\mathrm{PQ}}$ with the scale of electroweak symmetry breaking, implying an axion mass near the electron mass $m_{a} \approx \mathcal{O}\left(10^{2}\right) \mathrm{keV}$. However, experimental searches for such axions have so far been unsuccessful [7], and indeed only a narrow allowed window currently exists:

$$
10^{10} \mathrm{GeV} \lesssim f_{\mathrm{PQ}} \lesssim 10^{12} \mathrm{GeV} .
$$

This then implies an axion which is exceedingly light,

$$
10^{-5} \mathrm{eV} \leqslant m_{a} \lesssim 10^{-3} \mathrm{eV},
$$

and whose couplings to ordinary matter are exceedingly suppressed. These bounds generally result from various combinations of laboratory, astrophysical, and cosmological constraints. In all cases, however, the crucial ingredient is the correlation between the mass of the axion and the strength of its couplings to matter, since both are essentially determined by the single parameter $f_{\mathrm{PQ}}$.

In this paper, we point out that this situation may be drastically altered in theories with large extra spacetime dimensions. Since their original proposal [8], such theories have recently received considerable attention because of their prospects for lowering the fundamental grand unified theory (GUT) scale [9], the fundamental Planck scale [10], and the fundamental string scale [11]. As a result of these developments, it is now understood that all three of these scales may be adjusted to arbitrary values, perhaps even values in the $\mathrm{TeV}$ range, without violating experimental constraints. Of course, if one lowers these fundamental scales below the PQ symmetry-breaking scale in Eq. (6), then it might seem difficult to preserve the axion solution to the strong $C P$ problem. In fact, this observation has been used [12] to argue that the fundamental scales of physics should be taken at some intermediate scale near the PQ scale. However, this argument assumes that the PQ mechanism itself remains untouched by the presence of the extra large dimensions.

In this paper, we shall generalize the PQ mechanism to higher dimensions. More specifically, we shall consider the consequences of placing the PQ axion in the "bulk" (i.e., perpendicular to the $p$-brane that contains the standard model) so that the axion accrues an infinite tower of KaluzaKlein excitations. Placing the QCD axion in the bulk has also been discussed previously in Refs. [10,13], and is completely analogous to recent ideas concerning the placement of the right-handed neutrino in the bulk $[14,15]$ in order to lower the neutrino seesaw scale and obtain neutrino oscillations with light (or even vanishing [14]) neutrino masses.

At first sight, it may seem that Kaluza-Klein modes for the axion cannot possibly have a significant effect on axion phenomenology. After all, we do not generally expect Kaluza-Klein modes to play a significant role unless their masses are of the same order as the mass of the usual fourdimensional state. However, because extra spacetime dimensions off the brane can potentially be as large as a millimeter, the lightest corresponding Kaluza-Klein modes can have masses $\sim \mathcal{O}\left(10^{-4} \mathrm{eV}\right)$. Note, in particular, that this bound does not rely on choosing any particular mass scale for our underlying higher-dimensional theory, and likewise makes no assumption concerning the (an-)isotropy of the compactification. Instead, this bound comes directly from the current status of gravitational Cavendish experiments. Remarkably, however, this Kaluza-Klein bound falls directly within the allowed range (7) for the mass of the four-dimensional axion. It is precisely for this reason that it is important to study the effects that Kaluza-Klein axions can have on axion phenomenology.

Given this, we shall find that placing the axion in the bulk can have a number of surprising consequences for axion physics, all of which provide new ways of rendering the axion "invisible."

First, in Sec. II, we shall show that the mass of the axion can be decoupled from the PQ symmetry-breaking scale, and under certain circumstances is essentially set by the radius $R$ of the bulk:

$$
m_{a} \lesssim \mathcal{O}\left(R^{-1}\right)
$$

Thus, remarkably, the allowed range (7) for the axion mass is consistent with radii in the millimeter or sub-millimeter range. More importantly, however, this result implies that it is possible to adjust the PQ symmetry breaking scale independently of the axion mass in order to control the strength of the couplings of this axion to ordinary matter. This feature does not arise in four dimensions, and might be useful in order to provide an alternative explanation for an "invisible" axion. A detailed numerical discussion of this possibility will be presented in Sec. V.

Second, in Sec. III, we shall show that the presence of an infinite tower of Kaluza-Klein axion modes can induce the novel phenomenon of laboratory axion oscillations. These oscillations are completely analogous to laboratory neutrino oscillations, but we shall find that under certain circumstances they lead to a complete and rapid decoherence of the axion field. This implies that an axion, once produced in the laboratory, will "decohere" extremely rapidly. This is therefore a second higher-dimensional phenomenon that can contribute to the "invisibility" of the axion under certain circumstances.

Third, in Sec. III, we shall also discuss the role played by the excited axion Kaluza-Klein states in axion-mediated processes and decays, and propose several experimental methods of detecting their existence. These would therefore provide direct experimental tests of the higher-dimensional nature of the axion field. 
Finally, in Sec. IV, we shall show that under certain circumstances, the presence of the infinite tower of KaluzaKlein axion modes can actually accelerate the dissipation of the energy associated with cosmological relic axion oscillations. This implies a weakening of the usual fourdimensional cosmological relic oscillation bounds on the PQ symmetry-breaking scale, which in turn may permit axionmatter couplings to be suppressed even more strongly than in the usual four-dimensional case. This is therefore a third higher-dimensional feature leading to an "invisible", axion.

Together, these results suggest that "invisible" axions can emerge quite naturally within the context of higherdimensional theories with large-radius compactifications, and have significantly different phenomenologies than they do in four dimensions. Moreover, many of our results apply to bulk fields in general, and transcend the specific case of the axion. This is illustrated in Sec. VI, where we consider the consequences of extra dimensions for another hypothetical particle, the so-called standard-model dilaton.

\section{A HIGHER-DIMENSIONAL PECCEI-QUINN MECHANISM}

In order to generalize the Peccei-Quinn (PQ) mechanism to higher dimensions, we will assume that there exists a complex scalar field $\phi$ in higher dimensions which transforms under a global $U(1)_{\mathrm{PQ}}$ symmetry:

$$
\phi \rightarrow e^{i \Lambda} \phi
$$

This symmetry is assumed to be spontaneously broken by the bulk dynamics so that $\langle\phi\rangle=f_{\mathrm{PQ}} / \sqrt{2}$, where $f_{\mathrm{PQ}}$ is the energy scale associated with the breaking of the PQ symmetry. We thus write our complex scalar field $\phi$ in the form

$$
\phi \approx \frac{f_{\mathrm{PQ}}}{\sqrt{2}} e^{i a / f_{\mathrm{PQ}}}
$$

where $a$ is the Nambu-Goldstone boson (axion) field. If we concentrate on the case of five dimensions for concreteness, then the kinetic-energy term for the scalar field takes the form

$$
\mathcal{S}_{\mathrm{K} . \mathrm{E} .}=\int d^{4} x d y M_{s} \partial_{M} \phi^{*} \partial^{M} \phi=\int d^{4} x d y M_{s} \frac{1}{2} \partial_{M} a \partial^{M} a
$$

where $M_{s}$ is a fundamental mass scale (e.g., a type I string scale), and where we have neglected the contributions from the radial mode. Here $x^{\mu}$ are the coordinates of the four uncompactified spacetime dimensions, $y$ is the coordinate of the fifth dimension, and the $M$ spacetime index runs over all five dimensions: $x^{M} \equiv\left(x^{\mu}, y\right)$. Note that there is no mass term for the axion, as this would not be invariant under the $U(1)_{\mathrm{PQ}}$ transformation

$$
a \rightarrow a+f_{\mathrm{PQ}} \Lambda \text {. }
$$

Furthermore, as a result of the chiral anomaly, we will also assume a bulk-boundary coupling of the form

$$
\mathcal{S}_{\text {coupling }}=\int d^{4} x d y \frac{\xi}{f_{\mathrm{PQ}}} \frac{g^{2}}{32 \pi^{2}} a F_{a}^{\mu \nu} \widetilde{F}_{\mu \nu a} \delta(y)
$$

where $F_{\mu \nu a}$ is the (four-dimensional) QCD field strength describing the QCD gauge fields which are confined to a fourdimensional subspace (e.g., a D-brane) located at $y=0$, and where $\xi$ is a model-dependent quantity parametrizing the strength of the axion couplings to matter. Thus, our total effective five-dimensional axion action takes the form

$$
\mathcal{S}_{\mathrm{eff}}=\int d^{4} x d y\left[\frac{1}{2} M_{s} \partial_{M} a \partial^{M} a+\frac{\xi}{f_{\mathrm{PQ}}} \frac{g^{2}}{32 \pi^{2}} a F_{a}^{\mu \nu} \widetilde{F}_{\mu \nu a} \delta(y)\right] .
$$

While we have assumed that the spontaneously broken $U(1)_{\mathrm{PQ}}$ is parametrized by $f_{\mathrm{PQ}}$, one still has to address the fact that gravitational effects can also break the $U(1)_{\mathrm{PQ}}$ symmetry. In other words, gravitational interactions do not respect global symmetries. Ultimately, this can lead to a gravitational contribution to the axion mass which is not necessarily suppressed. In this paper, however, we will assume that the gravitational contributions to the axion mass are indeed suppressed, and that $U(1)_{\mathrm{PQ}}$ remains a valid symmetry even in the presence of gravitational effects. For example, such suppression might arise due to the suppression of gravitational interactions across a large bulk, discrete symmetries of the sort that might come from ScherkSchwarz compactifications, or other large-radius effects. Likewise, we point out that if our underlying higherdimensional theory is a string theory, then such a global PQ symmetry can emerge only as an effective symmetry of the low-energy effective string Lagrangian. This is because string theory does not provide continuous global symmetries.

In order to obtain an effective four-dimensional theory, our next step is to compactify the fifth dimension. For simplicity, we shall assume that this dimension is compactified on a $\mathbb{Z}_{2}$ orbifold of radius $R$ where the orbifold action is identified as $y \rightarrow-y$. This implies that the axion field will have a Kaluza-Klein decomposition of the form

$$
a\left(x^{\mu}, y\right)=\sum_{n=0}^{\infty} a_{n}\left(x^{\mu}\right) \cos \left(\frac{n y}{R}\right)
$$

where $a_{n}\left(x^{\mu}\right) \in \mathbb{R}$ are the Kaluza-Klein modes and where we have demanded that the axion field be symmetric under the $Z_{2}$ action (in order to have a light zero mode that we can identify with the usual four-dimensional axion).

In principle, we should also allow for the possibility that the axion field winds non-trivially around the extra compactified dimension, with winding number $w$. This possibility of winding arises because $a$ is really only an angular variable, as evident from Eq. (10), and would imply that we should introduce an extra term $w f_{\mathrm{PQ}} y / R$ into the mode expansion (15). Note, in particular, that such windings cannot be removed by global Peccei-Quinn transformations of the form (12). However, such a winding term would not be invariant under the orbifold symmetry $y \rightarrow-y$, and thus only the unwound configuration $w=0$ survives the orbifold projection. Even if we were to compactify the axion field on a circle 
rather than an orbifold, such a term would contribute only an overall additive constant to the resulting effective potential for the axion field, and would not change any of the subsequent physics. It is therefore sufficient to restrict our attention to the unwound configuration with $w=0$.

It is also interesting to note that with respect to the fourdimensional Kaluza-Klein axion modes $a_{n}$, the PecceiQuinn transformation (12) takes the form

$$
\begin{aligned}
& a_{0} \rightarrow a_{0}+f_{\mathrm{PQ}} \Lambda \\
& a_{k} \rightarrow a_{k} \text { for all } k>0 .
\end{aligned}
$$

Thus, we see that only $a_{0}$ serves as the true axion transforming under the PQ transformation, while the excited KaluzaKlein modes $a_{k}$ remain invariant.

Substituting Eq. (15) into Eq. (14) and integrating over the fifth dimension, we then obtain an effective fourdimensional Lagrangian density

$$
\begin{aligned}
\mathcal{L}_{\mathrm{eff}}= & \mathcal{L}_{\mathrm{QCD}}+\frac{1}{2} \sum_{n=0}^{\infty}\left(\partial_{\mu} a_{n}\right)^{2}-\frac{1}{2} \sum_{n=1}^{\infty} \frac{n^{2}}{R^{2}} a_{n}^{2} \\
& +\frac{\xi}{\hat{f}_{\mathrm{PQ}}} \frac{g^{2}}{32 \pi^{2}}\left(\sum_{n=0}^{\infty} r_{n} a_{n}\right) F_{a}^{\mu \nu} \widetilde{F}_{\mu \nu a}
\end{aligned}
$$

where

$$
r_{n} \equiv \begin{cases}1 & \text { if } n=0 \\ \sqrt{2} & \text { if } n>0\end{cases}
$$

Note that in order to obtain Eq. (17), we must individually rescale each of the Kaluza-Klein modes $a_{n}$ in order to ensure that they have canonically normalized kinetic-energy terms. It is this that produces the relative rescaling coefficients $r_{n}$ in Eq. (18). We have also defined $\hat{f}_{\mathrm{PQ}} \equiv\left(V M_{s}\right)^{1 / 2} f_{\mathrm{PQ}}$, where $V$ is the volume of our compactified space. For $\delta$ extra dimensions, this definition generalizes to $\hat{f}_{\mathrm{PQ}} \equiv\left(V M_{s}^{\delta}\right)^{1 / 2} f_{\mathrm{PQ}}$. For example, assuming a $\delta$-dimensional toroidal compactification implies $V=(2 \pi R)^{\delta}$, resulting in the relation

$$
\hat{f}_{\mathrm{PQ}} \equiv\left(2 \pi R M_{s}\right)^{\delta / 2} f_{\mathrm{PQ}} .
$$

Note that while $f_{\mathrm{PQ}}$ sets the overall mass scale for the breaking of the Peccei-Quinn symmetry, it is the volumerenormalized quantity $\hat{f}_{\mathrm{PQ}}$ that parametrizes the coupling between the axion and the gluons. In general, since $M_{s}$ $\gg R^{-1}$, we find that $\hat{f}_{\mathrm{PQ}} \gg f_{\mathrm{PQ}}$. Therefore, as pointed out in Ref. [10], this volume-renormalization of the brane-bulk coupling can be used to obtain sufficiently suppressed axion-gauge-field couplings even if $f_{\mathrm{PQ}}$ itself is taken to be relatively small. In other words, even if we demand that $\hat{f}_{\mathrm{PQ}}$ be in the approximate range $10^{8} \mathrm{GeV} \leq \hat{f}_{\mathrm{PQ}} \lesssim 10^{12} \mathrm{GeV}$, the fundamental Peccei-Quinn symmetry-breaking scale $f_{\mathrm{PQ}}$ can be substantially reduced, potentially all the way to the $\mathrm{TeV}$ range. This volume suppression is thus one higherdimensional way [10] of avoiding the need for a high fundamental Peccei-Quinn scale $f_{\mathrm{PQ}}$.
Two comments are important at this stage. First, in toroidally compactified higher-dimensional theories with reduced Planck scales, the higher-dimensional Planck scale $M_{*}$ and the usual four-dimensional Planck scale $M_{\text {Planck }}$ are related to each other via [10]

$$
M_{\text {Planck }}=\left(2 \pi R M_{*}\right)^{n / 2} M_{*}
$$

where $n$ is the total number of extra spacetime dimensions in the bulk. Identifying $M_{*} \sim M_{s}$, we therefore see that the $n$-dimensional volume factor $\left(2 \pi R M_{s}\right)^{n / 2}$ must already be adjusted in order to account for the difference between $M_{s}$ $\sim \mathcal{O}(\mathrm{TeV})$ and $M_{\text {Planck }} \sim \mathcal{O}\left(10^{19} \mathrm{GeV}\right)$. If we were to take $\delta=n$ for the current axion case, this would imply either that $\hat{f}_{\mathrm{PQ}} \sim M_{\text {Planck }}$ (which would presumably overclose the universe), or that $M_{s} \ll \mathcal{O}(\mathrm{TeV})$ (which would clearly violate current experimental bounds). Therefore, if we assume an isotropic compactification with all radii taken equal, we see that an intermediate scale $\hat{f}_{\mathrm{PQ}}$ can be generated via Eq. (19) only if we have $\delta<n$. In other words, under these assumptions, the axion must be restricted to a subspace of the full higher-dimensional bulk. This has already been pointed out in Ref. [13], and is analogous to similar restrictions that arise in the case of higher-dimensional neutrinos $[14,15]$. Note that such "partial-bulk" fields generically arise in type I string theory; a discussion of some of their phenomenological effects and collider signatures can be found in Ref. [16].

Our second comment concerns the relevance of the mass scale $\hat{f}_{\mathrm{PQ}}$ that is generated by this volume factor. Of course, it is apparent from Eq. (17) that $\hat{f}_{\mathrm{PQ}}$ (rather than $f_{\mathrm{PQ}}$ ) sets the scale for couplings between gauge fields and individual axion modes $a_{k}$. However, we have also seen in Eq. (17) that the gauge fields couple not to an individual axion mode $a_{k}$, but rather to the linear superposition

$$
a^{\prime} \equiv \frac{1}{\sqrt{N}} \sum_{n=0}^{n_{\max }} r_{n} a_{n}=\frac{1}{\sqrt{N}}\left(a_{0}+\sqrt{2} \sum_{n=1}^{n_{\max }} a_{n}\right)
$$

where

$$
N \equiv 1+2 n_{\max } .
$$

Here $n_{\max }$ is a cutoff, determined according to the underlying mass scale $M_{s}$ (which sets the limit of validity of our higherdimensional effective field theory ${ }^{1}$ ). Taking $n_{\max } \approx R M_{s}$ $\gg 1$, we then find that the axion-gluon coupling in Eq. (17) takes the form

$$
\frac{\sqrt{N}}{\hat{f}_{\mathrm{PQ}}} a^{\prime} F_{a}^{\mu \nu} \widetilde{F}_{\mu \nu a} \approx \frac{1}{\sqrt{\pi} f_{\mathrm{PQ}}} a^{\prime} F_{a}^{\mu \nu} \widetilde{F}_{\mu \nu a} .
$$

Thus it is actually $f_{\mathrm{PQ}}$, rather than $\hat{f}_{\mathrm{PQ}}$, that sets the scale for axion couplings involving the entire Kaluza-Klein linear su-

\footnotetext{
${ }^{1}$ Note that the heavy modes with masses of order $M_{s}$ can be treated within field theory along the lines discussed in Ref. [17], and within string theory along the lines discussed in Ref. [18].
} 
perposition $a^{\prime}$. In other words, the effects of the volume factor in Eq. (19) are cancel led by the normalization of the Kaluza-Klein linear superposition $a^{\prime}$. Of course, this is expected from the perspective of the higher-dimensional theory in which $f_{\mathrm{PQ}}$ is the only fundamental mass scale, and indeed this cancellation of the volume factor persists for any number of extra spacetime dimensions. Because we expect $f_{\mathrm{PQ}}$ $\ll \hat{f}_{\mathrm{PQ}}$ in scenarios with large extra spacetime dimensions, we see that axion couplings involving the linear superposition $a^{\prime}$ are relatively strong, and pose a serious threat to the invisibility of the higher-dimensional axion. We shall discuss how this problem may be overcome in subsequent sections.

Similar observations also apply for axion couplings to standard-model fermions. The invariance under the $U(1)_{\mathrm{PQ}}$ transformation $a \rightarrow a+f_{\mathrm{PQ}} \Lambda$ implies that axions can be at most derivatively coupled to fermions carrying a PQ charge. If we assume that these fermions are also restricted to the D-brane at $y=0$ (as would be the case for all standard-model fermions), then this axion-fermion coupling is restricted to take the form

$$
\mathcal{S}_{a \psi \psi} \sim \int d^{4} x \frac{1}{\hat{f}_{\mathrm{PQ}}}\left(\left.\partial_{\mu} a\right|_{y=0}\right)\left(\bar{\psi} \gamma^{\mu} \gamma^{5} \psi\right)
$$

Here $\left.a\right|_{y=0}$ is the full five-dimensional bulk axion field evaluated at $y=0$, and the bulk-brane coupling strength $\hat{f}_{\mathrm{PQ}}$ is defined in Eq. (19). Note that $\hat{f}_{\mathrm{PQ}}$ is the same volumerescaled axion decay constant that parametrizes the couplings to the gauge fields, with the volume factor emerging just as for the axion-gauge couplings. Using the KaluzaKlein decomposition (15), the action then becomes

$$
\begin{aligned}
\mathcal{S}_{a \psi \psi} & \sim \frac{1}{\hat{f}_{\mathrm{PQ}}} \sum_{n=0}^{\infty} r_{n} \int d^{4} x\left(\partial_{\mu} a_{n}\right)\left(\bar{\psi} \gamma^{\mu} \gamma^{5} \psi\right) \\
& \sim \frac{1}{f_{\mathrm{PQ}}} \int d^{4} x\left(\partial_{\mu} a^{\prime}\right)\left(\bar{\psi} \gamma^{\mu} \gamma^{5} \psi\right),
\end{aligned}
$$

and we see that once again the entire Kaluza-Klein linear superposition $a^{\prime}$ couples to the charged fermions. This is completely analogous to the situation in Eq. (17) for the gauge fields. It is important to stress that this need not have been the result from a purely four-dimensional perspective. Indeed, given the Peccei-Quinn transformation properties (16), we see that it is only the zero-mode $a_{0}$ which requires a derivative coupling to fermions; the other axion modes $a_{k}$ are $a$ priori free to have non-derivative couplings. It is therefore only the higher-dimensional structure of the axion field that forces all of the axion modes to have identical derivative couplings to charged fermions. Moreover, we see from Eq. (25) that while the mass scale for the couplings of individual Kaluza-Klein axion fermions to fermions is set by $\hat{f}_{\mathrm{PQ}}$, the mass scale for the coupling of the full linear superposition $a^{\prime}$ to fermions is set by $f_{\mathrm{PQ}}$.

Let us now proceed to verify that this higher-dimensional Peccei-Quinn mechanism still cancels the $C P$-violating phase, and use this to calculate the mass of the axion. Given the Lagrangian (17), we observe that $a^{\prime} \sim \sum_{n=0}^{\infty} r_{n} a_{n}$ serves as the overall quantity that parametrizes the size of $C P$ symmetry breaking. Applying the one-instanton dilute-gas approximation, it is straightforward to show that

$$
\left\langle F_{a}^{\mu \nu} \widetilde{F}_{\mu \nu a}\right\rangle=-\Lambda_{\mathrm{QCD}}^{4} \sin \left(\frac{\xi}{\hat{f}_{\mathrm{PQ}}} \sum_{n=0}^{\infty} r_{n} a_{n}+\bar{\Theta}\right) .
$$

This gives rise to an effective potential for the axion modes in the QCD vacuum

$$
\begin{aligned}
V\left(a_{n}\right)= & \frac{1}{2} \sum_{n=1}^{\infty} \frac{n^{2}}{R^{2}} a_{n}^{2}+\frac{g^{2}}{32 \pi^{2}} \Lambda_{\mathrm{QCD}}^{4} \\
& \times\left[1-\cos \left(\frac{\xi}{\hat{f}_{\mathrm{PQ}}} \sum_{n=0}^{\infty} r_{n} a_{n}+\bar{\Theta}\right)\right] .
\end{aligned}
$$

In order to exhibit the Peccei-Quinn mechanism, we now minimize the axion effective potential,

$$
\frac{\partial V}{\partial a_{n}}=\frac{n^{2}}{R^{2}} a_{n}+r_{n} \frac{\xi}{\hat{f}_{\mathrm{PQ}}} \frac{g^{2}}{32 \pi^{2}} \Lambda_{\mathrm{QCD}}^{4} \sin \left(\frac{\xi}{\hat{f}_{\mathrm{PQ}}} \sum_{n=0}^{\infty} r_{n} a_{n}+\bar{\Theta}\right)=0
$$

yielding the unique solution

$$
\begin{aligned}
& \left\langle a_{0}\right\rangle=\frac{\hat{f}_{\mathrm{PQ}}}{\xi}(-\bar{\Theta}+l \pi), \quad l \in 2 \mathbb{Z} \\
& \left\langle a_{k}\right\rangle=0 \quad \text { for all } k>0 .
\end{aligned}
$$

Note that while any value $l \in \mathbb{Z}$ provides an extremum of the potential, only the values $l \in 2 \mathbb{Z}$ provide the desired minimum of the potential. Thus, this higher-dimensional Peccei-Quinn mechanism continues to solve the strong $C P$ problem: we see that $a_{0}$ is the usual Peccei-Quinn axion which solves the strong $C P$ problem by itself by cancelling the $\bar{\Theta}$ angle, while all of the excited Kaluza-Klein axions $a_{k}$ for $k>0$ have vanishing VEVs. This makes sense, since only $a_{0}$ is a true massless Nambu-Goldstone field from the fourdimensional perspective of Eq. (17). This is also evident from the Peccei-Quinn transformation properties (16).

However, as we shall now show, these excited KaluzaKlein axion states nevertheless have a drastic effect on the axion mass matrix. In order to derive the mass matrix, we consider the local curvature of the effective axion potential around its minimum:

$$
\left.\mathcal{M}_{n n^{\prime}}^{2} \equiv \frac{\partial^{2} V}{\partial a_{n} \partial a_{n^{\prime}}}\right|_{\langle a\rangle}
$$

From this we obtain 


$$
\begin{aligned}
\mathcal{M}_{n n^{\prime}}^{2} \equiv & \frac{n^{2}}{R^{2}} \delta_{n n^{\prime}}+\xi^{2} \frac{g^{2}}{32 \pi^{2}} \frac{\Lambda_{\mathrm{QCD}}^{4}}{\hat{f}_{\mathrm{PQ}}^{2}} r_{n} r_{n^{\prime}} \\
& \times\left.\cos \left(\frac{\xi}{\hat{f}_{\mathrm{PQ}}} \sum_{n=0}^{\infty} r_{n} a_{n}+\bar{\Theta}\right)\right|_{\langle a\rangle},
\end{aligned}
$$

and in the vicinity of the minimum (29) this becomes

$$
\mathcal{M}_{n n^{\prime}}^{2}=\frac{n^{2}}{R^{2}} \delta_{n n^{\prime}}+\xi^{2} \frac{g^{2}}{32 \pi^{2}} \frac{\Lambda_{\mathrm{QCD}}^{4}}{\hat{f}_{\mathrm{PQ}}^{2}} r_{n} r_{n^{\prime}} .
$$

Let us now define

$$
\begin{aligned}
m_{\mathrm{PQ}}^{2} & \equiv \xi^{2} \frac{g^{2}}{32 \pi^{2}} \frac{\Lambda_{\mathrm{QCD}}^{4}}{\hat{f}_{\mathrm{PQ}}^{2}} \\
y & \equiv \frac{1}{m_{\mathrm{PQ}} R} .
\end{aligned}
$$

Thus $m_{\mathrm{PQ}}$ is the expected mass that the axion would ordinarily have taken in four dimensions (depending on $\hat{f}_{\mathrm{PQ}}$ rather than $f_{\mathrm{PQ}}$ itself), and $y$ is the ratio of the scale of the extra dimension to $m_{\mathrm{PQ}}$. Our mass matrix then takes the form

$$
\mathcal{M}^{2}=m_{\mathrm{PQ}}^{2}\left(r_{n} r_{n^{\prime}}+y^{2} n^{2} \delta_{n n^{\prime}}\right)
$$

or equivalently

$$
\mathcal{M}^{2}=m_{\mathrm{PQ}}^{2}\left(\begin{array}{ccccc}
1 & \sqrt{2} & \sqrt{2} & \sqrt{2} & \cdots \\
\sqrt{2} & 2+y^{2} & 2 & 2 & \cdots \\
\sqrt{2} & 2 & 2+4 y^{2} & 2 & \ldots \\
\sqrt{2} & 2 & 2 & 2+9 y^{2} & \ldots \\
\vdots & \vdots & \vdots & \vdots & \ddots
\end{array}\right) .
$$

Note that the usual Peccei-Quinn case corresponds to the upper-left $1 \times 1$ matrix, leading to the expected result $\mathcal{M}^{2}$ $=m_{\mathrm{PQ}}^{2}$. Thus, the additional rows and columns reflect the extra Kaluza-Klein states, and their physical effect is to pull the lowest eigenvalue of this matrix away from $m_{\mathrm{PQ}}^{2}$.

Deriving the condition for the eigenvalues of this matrix is straightforward. Let us denote the eigenvalues of this matrix as $\lambda^{2}$ rather than $\lambda$ because this is a (mass) ${ }^{2}$ matrix. We then find that the eigenvalues are given as the solutions to the transcendental equation

$$
\frac{\pi \tilde{\lambda}}{y} \cot \left(\frac{\pi \tilde{\lambda}}{y}\right)=\tilde{\lambda}^{2}
$$

where we have defined the dimensionless eigenvalue

$$
\tilde{\lambda} \equiv \lambda / m_{\mathrm{PQ}}
$$

In terms of dimensionful quantities, this transcendental equation takes the equivalent form ${ }^{2}$

$$
\pi R \lambda \cot (\pi R \lambda)=\frac{\lambda^{2}}{m_{\mathrm{PQ}}^{2}} .
$$

For each eigenvalue $\lambda$, the corresponding normalized mass eigenstate $\hat{a}_{\lambda}$ is exactly given by

$$
\hat{a}_{\lambda}=\sum_{k=0}^{\infty} U_{\lambda k} a_{k}
$$

where $a_{k}$ are the Kaluza-Klein axion modes given in Eq. (15) and where $U_{\lambda k}$ is the unitary matrix that diagonalizes $\mathcal{M}^{2}$. This matrix is given by

$$
U_{\lambda k} \equiv\left(\frac{r_{k} \tilde{\lambda}^{2}}{\widetilde{\lambda^{2}-k^{2} y^{2}}}\right) A_{\lambda}
$$

where

$$
A_{\lambda} \equiv \frac{\sqrt{2}}{\tilde{\lambda}}\left(\tilde{\lambda}^{2}+1+\pi^{2} / y^{2}\right)^{-1 / 2}
$$

Note that the unitarity of the matrix $U$ implies that $\Sigma_{\lambda}\left|U_{\lambda 0}\right|^{2}=1$, which in turn implies

$$
\sum_{\lambda} A_{\lambda}^{2}=1
$$

For future reference, we also record another useful identity which will be proven in Sec. IV:

$$
\sum_{\lambda} \tilde{\lambda}^{2} A_{\lambda}^{2}=1
$$

Finally, combining Eqs. (36) and (40), it is straightforward to show that

\footnotetext{
${ }^{2}$ Interestingly, this eigenvalue equation is identical to that which emerges [14] when the right-handed neutrino $\nu_{R}$ is placed in the bulk, with the mass scale $m_{\mathrm{PQ}}$ in the axion case corresponding to the Dirac coupling $m$ in the neutrino case. This implies that there is a formal relation between the Kaluza-Klein axion modes and the Kaluza-Klein neutrino modes. Remarkably, this correspondence exists even though the axion and right-handed neutrino have different spins, and even though the mechanisms for mass generation are completely different in the two cases. Moreover, in Sec. VI, we shall demonstrate that the same mass matrix and eigenvalue equation also emerge when the standard-model dilaton is placed in the bulk of extra spacetime dimensions. This suggests that many of the higher-dimensional phenomena to be discussed in this paper (such as laboratory and cosmological relic axion oscillations) may have a correspondingly general phenomenology that is equally applicable to neutrinos, dilatons, as well as other bulk fields that transform as singlets under the standard-model gauge group.
} 


$$
\sum_{k=0}^{\infty} r_{k} U_{\lambda k}=\widetilde{\lambda}^{2} A_{\lambda} .
$$

Note that all three of these identities hold for all values of $y \equiv\left(m_{\mathrm{PQ}} R\right)^{-1}$. This in turn allows us to rewrite Eq. (21) in the form

$$
a^{\prime}=\frac{1}{\sqrt{N}} \sum_{\lambda} \tilde{\lambda}^{2} A_{\lambda} \hat{a}_{\lambda}
$$

We can check that Eq. (38) makes sense in the limit $R \rightarrow 0$. In this limit, the Kaluza-Klein states become infinitely heavy and decouple; thus we should be left with the lightest eigenvalue $\lambda=m_{\mathrm{PQ}}$. And this is indeed what happens: as $R \rightarrow 0$, we see $\pi R \lambda \cot (\pi R \lambda) \rightarrow 1$, whereupon we obtain $\lambda=m_{\mathrm{PQ}}$, with all other eigenvalues infinitely heavy. As $R$ becomes larger, the effect of the extra large dimension is felt through a reduction of this lowest eigenvalue. Thus, as $R$ increases, the mass of the lightest axion decreases.

One important consequence of Eq. (38) is that the lightest axion mass eigenvalue $m_{a}$ is strictly bounded by the radius

$$
m_{a} \leqslant \frac{1}{2} R^{-1} \text {. }
$$

This result (46) holds regardless of the value of $m_{\mathrm{PQ}}$. Thus, in higher dimensions, we see that when $m_{\mathrm{PQ}} \gtrsim \frac{1}{2} R^{-1}$, the size of the axion mass is set by the radius $R$ and not by the Peccei-Quinn scale $f_{\mathrm{PQ}}$. In Fig. 1 we show the value of the axion mass $m_{a}$ as a function of $y^{-1} \equiv m_{\mathrm{PQ}} R$. Of course, for $m_{\mathrm{PQ}} R \rightarrow 0$ (corresponding to either $R \rightarrow 0$ or $m_{\mathrm{PQ}} \rightarrow 0$ ), we see that we indeed have the expected result $m_{a} \approx m_{\mathrm{PQ}}$. This is indicated by the diagonal dashed line in Fig. 1. However, as $m_{\mathrm{PQ}} R$ increases, we see that the axion mass departs from this expected linear behavior, and instead is bounded by the inverse radius of the extra spacetime dimension. In fact, from Fig. 1, we see that we can approximate the mass of the axion as

$$
m_{a} \approx \min \left(\frac{1}{2} R^{-1}, m_{\mathrm{PQ}}\right)
$$

Thus the mass of the axion is determined solely by the radius of the extra spacetime dimension when $\frac{1}{2} R^{-1} \lesssim m_{\mathrm{PQ}}$.

As a result of this unexpected higher-dimensional behavior for the axion mass, we see that when $m_{\mathrm{PQ}} \gtrsim \frac{1}{2} R^{-1}$, the Peccei-Quinn scale $f_{\mathrm{PQ}}$ essentially decouples from the axion mass. Indeed, as long as $m_{\mathrm{PQ}} \gtrsim \frac{1}{2} R^{-1}$, we see that $m_{a}$ $\leqslant \frac{1}{2} R^{-1}$ regardless of the specific sizes of $m_{\mathrm{PQ}}$ or $\Lambda_{\mathrm{QCD}}$.

This observation has a number of interesting implications. First, given Eq. (46), we see that an axion mass in the allowed range (7) is already achieved for $R$ in the submillimeter range, independently of $m_{\mathrm{PQ}}$. This therefore provides further motivation for such submillimeter extra dimensions.

Second, and even more importantly, this result implies that the usual relation between the mass of the axion and its couplings to ordinary matter no longer holds in higher dimensions. Indeed, we have the surprising result that $f_{\mathrm{PQ}}$ can still be lowered or raised arbitrarily without affecting the axion mass, provided $m_{\mathrm{PQ}} \gtrsim \frac{1}{2} R^{-1}$. This suggests that it may

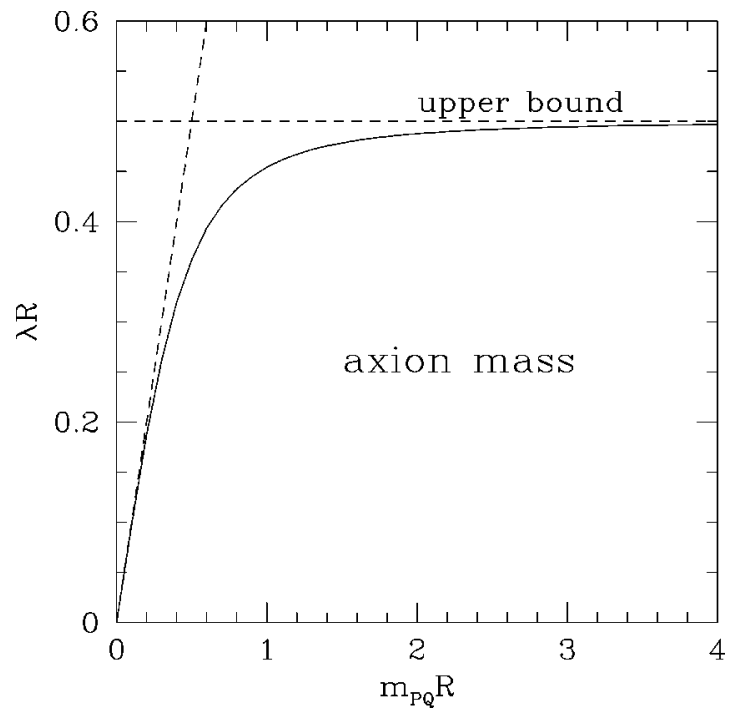

FIG. 1. The mass of the axion zero mode as a function of the dimensionless product $m_{\mathrm{PQ}} R \equiv y^{-1}$, where $m_{\mathrm{PQ}} \sim \Lambda_{\mathrm{QCD}}^{2} / \hat{f}_{\mathrm{PQ}}$. Although this reproduces the expected result $m_{a} \approx m_{\mathrm{PQ}}$ when $m_{\mathrm{PQ}}$ $\ll R^{-1}$ (corresponding to the diagonal dashed line $\lambda R=m_{\mathrm{PQ}} R$ ), we see that the axion mass is strictly bounded by the inverse radius in higher dimensions, with the precise value of $m_{\mathrm{PQ}}$ essentially decoupling for $m_{\mathrm{PQ}} \gtrsim \frac{1}{2} R^{-1}$. This implies that in higher dimensions, the Peccei-Quinn scale $f_{\mathrm{PQ}}$ can be adjusted arbitrarily within this parameter range without affecting the axion mass.

be possible to tune the fundamental Peccei-Quinn symmetry breaking scale $f_{\mathrm{PQ}}$ in such a way as to weaken the axion couplings to matter to whatever values are required to make the axion sufficiently invisible, all without affecting the axion mass. This may therefore provide a new method of obtaining an invisible axion. As we shall see, however, this possibility is subject to several important numerical constraints. We shall therefore defer a detailed discussion of this possibility to Sec. V.

\section{LABORATORY AXION OSCILLATIONS}

In this section we discuss the novel possibility of laboratory axion oscillations. Note that these are not the traditional cosmological relic axion oscillations (which will be discussed in Sec. IV), but rather "laboratory" axion oscillations that now arise in higher dimensions because the physical Peccei-Quinn axion $a_{0}$ is no longer a mass eigenstate. These laboratory axion oscillations are therefore completely analogous to laboratory neutrino oscillations, which similarly arise because the neutrino gauge eigenstates differ from the neutrino mass eigenstates. In the present axion case, such oscillations are possible because of the non-diagonal nature of the axion mass matrix (35). Therefore, as it propagates, it is possible for the four-dimensional axion zero-mode $a_{0}$ to oscillate into any of the higher-frequency axion modes in its Kaluza-Klein tower. Indeed, as we shall see, these oscillations can provide yet another mechanism that may contribute to the "invisibility" of the axion. 

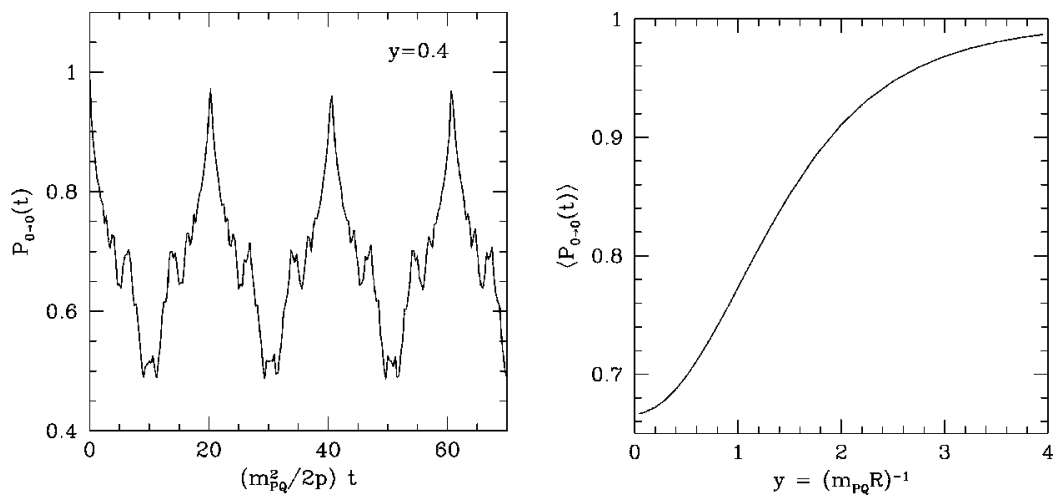

FIG. 2. Higher-dimensional axion oscillations, as discussed in the text. (a) The probability $P_{0 \rightarrow 0}(t)$ that the axion zero mode is preserved as a function of time, taking a reference value $y$ $\equiv\left(m_{\mathrm{PQ}} R\right)^{-1}=0.4$. (b) The period-averaged preservation probability $\left\langle P_{0 \rightarrow 0}(t)\right\rangle$ as a function of $y$. The limit $y \gg 1$ corresponds to the usual fourdimensional case, while the opposite $y \ll 1$ limit corresponds to an extremely large extra dimension with very light Kaluza-Klein states.

\section{A. Laboratory oscillations of the axion zero mode $a_{0}$}

It is straightforward to calculate these oscillation probabilities in terms of the mass mixing matrix (35) and the $U$-matrix (40) that diagonalizes it. From a four-dimensional perspective, we see from Eq. (16) that only the zero mode $a_{0}$ serves as a bonafide axion transforming under the PQ transformation. Therefore, let us first calculate the probability that this four-dimensional axion $a_{0}$ oscillates into any of its corresponding Kaluza-Klein excitations as it propagates, or conversely the probability that the four-dimensional axion $a_{0}$ is preserved as a function of time. Assuming that the axion is given an initial highly relativistic momentum $p$, we find that the probability that $a_{0}$ is preserved is given by

$$
\begin{aligned}
P_{0 \rightarrow 0}(t)= & \left|\sum_{\lambda} A_{\lambda}^{2} e^{-\Gamma_{\lambda} t / 2} \exp \left(\frac{i \lambda^{2} t}{2 p}\right)\right|^{2} \\
= & \sum_{\lambda} A_{\lambda}^{4} e^{-\Gamma_{\lambda} t+2 \sum_{\lambda^{\prime}<\lambda} A_{\lambda}^{2} A_{\lambda^{\prime}}^{2} e^{-\left(\Gamma_{\lambda}+\Gamma_{\lambda^{\prime}}\right) t / 2}} \\
& \times \cos \left(\frac{\left[\lambda^{2}-\left(\lambda^{\prime}\right)^{2}\right] t}{2 p}\right) .
\end{aligned}
$$

Here $A_{\lambda}$ is defined in Eq. (41), and $\Gamma_{\lambda}$ is the decay width of the corresponding Kaluza-Klein axion. Thus, even though $P_{0 \rightarrow 0}=1$ at the initial time $t=0$, we see that this probability decreases at later times and ultimately oscillates around a period-averaged value

$$
\left\langle P_{0 \rightarrow 0}(t)\right\rangle=\sum_{\lambda} A_{\lambda}^{4} e^{-\Gamma_{\lambda} t}
$$

which itself diminishes exponentially with time. Note that the calculations leading to these results are similar to the higher-dimensional neutrino oscillation calculations in Ref. [14].

It is important at this stage to separate two effects which influence the axion preservation probability. The first is the oscillation itself, which arises due to the non-trivial axion mass matrix and which reflects the mixing of the excited Kaluza-Klein axion states. It is this oscillation which is our focus in this section. By contrast, the second effect is axion decay as reflected in the decay widths $\Gamma_{\lambda}$. In general, these decay widths result from the dominant decay mode $\hat{a}_{\lambda}$ $\rightarrow \gamma \gamma$, and therefore scale as $\Gamma_{\lambda} \approx \lambda^{3} / \hat{f}_{\mathrm{PQ}}^{2}$. This implies that for the lowest Kaluza-Klein eigenvalues and sufficiently small times, the product $\Gamma_{\lambda} t$ is typically extremely small. This is in accordance with our expectation that the usual four-dimensional axion is extremely stable. For example, taking $\lambda_{0} \approx m_{\mathrm{PQ}} \approx 10^{-5} \mathrm{eV}$, we see that $\Gamma_{0} t \ll 1$ for all times $t \lesssim 10^{48} \mathrm{sec}$. This upper limit exceeds the age of the universe by 20 orders of magnitude. Therefore, particularly for the lowest Kaluza-Klein eigenvalues, it is safe to neglect these decay widths entirely, and concentrate solely on the oscillations. For example, since $A_{\lambda}^{4}$ decreases rapidly as a function of $\lambda$, only the lowest eigenvalues dominate the sum in Eq. (49). We therefore find that we can approximate

$$
\left\langle P_{0 \rightarrow 0}(t)\right\rangle \approx \sum_{\lambda} A_{\lambda}^{4}
$$

which is independent of time. We stress, however, that the decay widths $\Gamma_{\lambda}$ grow rapidly as a function of the mass of the Kaluza-Klein eigenstate $\hat{a}_{\lambda}$. We have therefore included these decay widths in Eqs. (48) and (49) for completeness, and will discuss the effects that they induce more carefully at the end of this section.

In Fig. 2(a), we have plotted the behavior of $P_{0 \rightarrow 0}(t)$ as a function of time, taking a reference value $y \equiv\left(m_{\mathrm{PQ}} R\right)^{-1}$ $=0.4$. Note that the jaggedness of the probability curve reflects the multi-component nature of the oscillation in which many different individual Kaluza-Klein oscillations interfere with incommensurate phases. Although this oscillation clearly leads to both axion deficits and axion regenerations, we see that while the axion regenerations are nearly total, the axion deficits are not total. This is in marked contrast to the results from a simple two-state oscillation. We also observe that these oscillations are approximately periodic, with a wavelength set by the lowest-lying eigenvalue difference. This is because it is the lowest-lying Kaluza-Klein axions that play the dominant role in producing this oscillation. As a result of this fact, we see that $\left\langle P_{0 \rightarrow 0}(t)\right\rangle$ is effectively constant as a function of time, in accordance with Eq. (50). Indeed, the interpretation of this oscillation is completely analogous to that given in Ref. [14] for higher-dimensional neutrino oscillations. We shall discuss the possibilities for experimentally detecting such oscillations at the end of this section.

In Fig. 2(b), we have plotted the time-averaged probability $\left\langle P_{0 \rightarrow 0}(t)\right\rangle$ given in Eq. (50) as a function of $y$ 
$\equiv\left(m_{\mathrm{PQ}} R\right)^{-1}$. For $y \gg 1$ (corresponding to the usual fourdimensional limit), we see that $\left\langle P_{0 \rightarrow 0}(t)\right\rangle \rightarrow 1$, as expected, reflecting the fact that our single axion field cannot oscillate because its Kaluza-Klein states are infinitely heavy and essentially decouple. More interestingly, however, we see that in the $y \ll 1$ limit (corresponding to extremely large radii or equivalently a quasi-continuous spectrum of light KaluzaKlein modes), the oscillation probability (50) approaches a fixed value

$$
\lim _{y \rightarrow 0}\left\langle P_{0 \rightarrow 0}(t)\right\rangle=\lim _{y \rightarrow 0} \sum_{\lambda} A_{\lambda}^{4}=\frac{2}{3} .
$$

Thus, for extremely large radii, we expect to see on average only $2 / 3$ of the axion flux that would have appeared in the four-dimensional case.

\section{B. Laboratory oscillations of the axion superposition $a^{\prime}$}

For many practical purposes, there exists a different probability that may be more relevant as a measure of laboratory axion oscillations. As we have seen in Sec. II, standardmodel gauge bosons and fermions generically couple not to $a_{0}$, but rather to the linear superposition $a^{\prime}$ given in Eq. (21). Thus, in any laboratory process that produces axions or is mediated by axions, a more crucial oscillation probability is the probability $P_{a^{\prime} \rightarrow a^{\prime}}(t)$ that this particular linear combination $a^{\prime}$ is preserved as a function of time. Indeed, we have already seen in Sec. II that while the standard-model couplings to individual axion modes scale as $1 / \hat{f}_{\mathrm{PQ}}$ and hence are already somewhat "invisible," the couplings to $a^{\prime}$ scale as $1 / f_{\mathrm{PQ}}$ and hence are significantly larger. Such couplings therefore pose the largest immediate threat to axion invisibility.

The calculation of the probability $P_{a^{\prime} \rightarrow a^{\prime}}(t)$ proceeds in an analogous manner. In general, the amplitude for an axion transition $a_{k} \rightarrow a_{l}$ is given by

$$
A_{k \rightarrow l}(t)=\sum_{\lambda} U_{\lambda l} U_{\lambda k}^{*} e^{-\Gamma_{\lambda} t / 2} e^{-i \lambda^{2} t / 2 p}
$$

where $U_{\lambda k}$ are the (real) unitary matrix elements defined in Eq. (40) and where we have omitted an overall $(k, l)$-independent phase. The probability that $a^{\prime}$ is preserved as a function of time is therefore given by

$$
P_{a^{\prime} \rightarrow a^{\prime}}(t)=\frac{1}{N^{2}}\left|\sum_{k, l=0}^{\infty} r_{k} r_{l} A_{k \rightarrow l}(t)\right|^{2}
$$

where the normalization factor $N$ is given in Eq. (22). In evaluating Eq. (53), it is convenient to use the identity (44) in order to perform the Kaluza-Klein summations. We thus find

$$
\begin{aligned}
P_{a^{\prime} \rightarrow a^{\prime}}(t)= & \frac{1}{N^{2}}\left[\sum_{\lambda}{\tilde{\lambda^{8}}}_{\lambda}^{4} e^{-\Gamma_{\lambda} t}\right. \\
& +2 \sum_{\lambda^{\prime}<\lambda}{\tilde{\lambda^{4}}}_{\left(\tilde{\lambda}^{\prime}\right)^{4} A_{\lambda}^{2} A_{\lambda^{\prime}}^{2} e^{-\left(\Gamma_{\lambda}+\Gamma_{\lambda^{\prime}}\right) t / 2}} \\
& \left.\times \cos \left(\frac{\left[\lambda^{2}-\left(\lambda^{\prime}\right)^{2}\right] t}{2 p}\right)\right],
\end{aligned}
$$

implying a period-averaged probability

$$
\left\langle P_{a^{\prime} \rightarrow a^{\prime}}(t)\right\rangle=\frac{1}{N^{2}} \sum_{\lambda} \tilde{\lambda}^{8} A_{\lambda}^{4} e^{-\Gamma_{\lambda} t} .
$$

Just as with $P_{0 \rightarrow 0}(t)$, we will find it convenient to distinguish between two different effects: the overall "damping", that arises due to axion decays (encoded within the decay widths $\Gamma_{\lambda}$ ), and the oscillations that arise due to the nontrivial mixings of the excited Kaluza-Klein states. In order to concentrate on the latter effect, we shall therefore set $\Gamma_{\lambda}$ $=0$ for simplicity. Moreover, as we shall see, this assumption will not change our phenomenological results regardless of the time interval $t$ in Eq. (57). Thus, taking $\Gamma_{\lambda}=0$, we see that Eq. (54) reduces to

$$
\begin{aligned}
P_{a^{\prime} \rightarrow a^{\prime}}(t)= & \frac{1}{N^{2}}\left[\sum_{\lambda} \tilde{\lambda}^{8} A_{\lambda}^{4}+2 \sum_{\lambda^{\prime}<\lambda} \tilde{\lambda}^{4}\left(\tilde{\lambda}^{\prime}\right)^{4} A_{\lambda}^{2} A_{\lambda^{\prime}}^{2}\right. \\
& \left.\times \cos \left(\frac{\left[\lambda^{2}-\left(\lambda^{\prime}\right)^{2}\right] t}{2 p}\right)\right],
\end{aligned}
$$

implying a time-averaged probability

$$
\left\langle P_{a^{\prime} \rightarrow a^{\prime}}(t)\right\rangle=\frac{1}{N^{2}} \sum_{\lambda} \tilde{\lambda}^{8} A_{\lambda}^{4} .
$$

It is straightforward to evaluate this time-averaged probability for different values of $y$. Remarkably, however, we find that

$$
\lim _{n_{\max } \rightarrow \infty}\left\langle P_{a^{\prime} \rightarrow a^{\prime}}(t)\right\rangle=0 \text { ! }
$$

We therefore have virtually no probability for detecting the linear combination $a^{\prime}$ at any later time after it is produced. Indeed, at the initial time $t=0$, the axion probability starts at 1. This is guaranteed by the unitarity of the $U$-matrix in Eq. (40), and may be verified directly from Eq. (56). However, for $t>0$, the multi-component Kaluza-Klein oscillations drive the net probability rapidly to zero. Indeed, as we shall see, this holds for all $t>0$, and does not rely on taking the $t \rightarrow \infty$ limit. At no later time does a macroscopic axion regeneration appear. It is for this reason that it is justified to set $\Gamma_{\lambda}=0$ in Eqs. (54) and (55).

Of course, these conclusions rely on taking the $n_{\max } \rightarrow \infty$ limit in Eq.(58). However, even if we truncate $n_{\max }$ at a finite value $\sim \mathcal{O}\left(M_{\text {string }} R\right)$ (reflecting the expected limit of validity 
for our effective field-theoretic treatment), we find

$$
\left\langle P_{a^{\prime} \rightarrow a^{\prime}}(t)\right\rangle \lesssim\left(M_{\text {string }} R\right)^{-1} .
$$

For $M_{\text {string }} \approx 10 \mathrm{TeV}$ and $R \approx 1$ millimeter, this implies

$$
\left\langle P_{a^{\prime} \rightarrow a^{\prime}}(t)\right\rangle \lesssim \mathcal{O}\left(10^{-17}\right) .
$$

Such an axion-mediated process therefore continues to be truly invisible in the sense that the $a^{\prime}$ state literally "disappears" over a very short time interval, with only a vanishingly small probability for detection of the $a^{\prime}$ state at any later time. This behavior is illustrated in Fig. 3. As we have noted, this is important because the $a^{\prime}$ state couples to standard-model fields with an unsuppressed coupling $1 / f_{\mathrm{PQ}}$ rather than with a volume-suppressed coupling $1 / \hat{f}_{\mathrm{PQ}}$.

Note that as $y \rightarrow \infty$ (reproducing the four-dimensional limit), the time needed for the probability to drop to zero increases without bound. Thus, in the four-dimensional limit $y \rightarrow \infty$, the probability begins and remains at 1 , as expected. However, for all finite values of $y$, the axion probability $P_{a^{\prime} \rightarrow a^{\prime}}(t)$ drops to zero in finite time, and remains there (as shown in Fig. 3). Indeed, for $n_{\max } \gg 1$, we see that the time $\tau_{0}$ for the axion probability to drop to a predetermined fraction of its initial value scales as ${ }^{3}$

${ }^{3}$ Note that the derivations of these scaling results can often be quite subtle. In order to derive Eq. (62), we first observe that $\tau_{0}$ $\sim t_{0} / N^{2}$, where $N$ is the normalization factor in Eq. (22) and where $t_{0}$ is a time scale that is independent of $n_{\max }$. This scaling behavior holds independently of $y$ when $n_{\max } \gg 1$, as can be verified numerically. Given this, it is straightforward to investigate the behavior of $t_{0}$ as a function of $y$, leading to the result $t_{0} \sim y^{-2}$. We thus obtain $\tau_{0} \sim\left(n_{\max } y\right)^{-2}$. The final step is to realize that there is a hidden $y$-dependence buried in the meaning of the cutoff $n_{\max }$, and that in order to compare cutoffs for different values of $y$, we must choose a uniform $y$-independent convention for the Kaluza-Klein truncation. Specifically, for each value of $y$, we must choose an appropriate $y$-dependent normalization of the cutoff $n_{\max }$ such that the rescaled cutoff $n_{\max }^{\prime}$ has a fixed, $y$-independent net effect in an eigenvalue sum such as Eq. (56). It turns out that such a renormalization compels us to choose $n_{\max }^{\prime} \sim y^{2} n_{\max }$. We therefore find $\tau_{0}$ $\sim y^{2} /\left(n_{\max }^{\prime}\right)^{2}$. To provide some explicit numbers, let us define $\tau_{0}$ as the time needed for the axion probability to fall to $10 \%$ of its initial value, and let us likewise define $n_{\text {crit }}$ to be the minimum number of Kaluza-Klein eigenvalues that must be included in the sum in Eq. (56) order to produce an initial axion probability of 0.99 . (In this connection, note that it is only in the formal $n_{\max } \rightarrow \infty$ limit that the initial probability truly approaches 1 .) We then find that $t_{0}$ $\approx 9.665 / y^{2}$ and $n_{\text {crit }} \approx 981 / y^{2}$. This latter relation enables us to normalize our values of $n_{\max }$ in relation to $n_{\text {crit }}$ by defining $n_{\max }^{\prime}$ $\equiv n_{\text {max }} / n_{\text {crit }}=y^{2} n_{\text {max }} / 981$, leading to the final result

$$
\left(\frac{m_{\mathrm{PQ}}^{2}}{2 p}\right)_{0} \approx 10^{-5} \frac{y^{2}}{\left(n_{\max }^{\prime}\right)^{2}} .
$$

The value of the rescaled cutoff $n_{\max }^{\prime}$ is then arbitrary, and may be chosen according to considerations beyond those of our effective field-theory approach (such as truncating according to the underlying string scale).

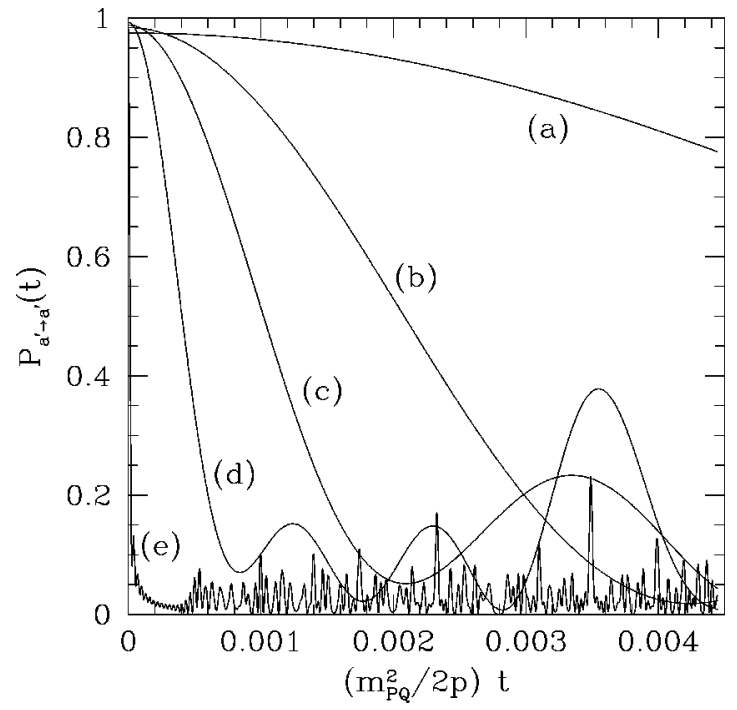

FIG. 3. The axion preservation probability $P_{a^{\prime} \rightarrow a^{\prime}}(t)$ as a function of the number $n_{\max }$ of Kaluza-Klein states which are included in the system. For this plot we have set $y=15$, and taken (a) $n_{\max }$ $=1$; (b) $n_{\max }=2$; (c) $n_{\max }=3$; (d) $n_{\max }=5$; and (e) $n_{\max }=30$. As $n_{\max }$ increases, the axion probability rapidly falls to zero as a result of the destructive interference of the Kaluza-Klein states, and remains suppressed without significant axion regeneration at any later times. Note, in particular, that the "spikes" in this plot are also suppressed as $\sim 1 / n_{\max }$, and vanish for large $n_{\max }$. Thus, for significantly large $n_{\max }$, the destructive interference of the excited Kaluza-Klein states causes the axion to "decohere," implying that there is negligible probability for subsequently detecting the original axion state at any future time.

$$
\tau_{0} \sim \frac{y^{2}}{n_{\max }^{2}} .
$$

Thus, as $y \rightarrow \infty$, we see that $\tau_{0} \rightarrow \infty$, while for finite values of $y$ this "decoherence time" is extremely short. This results in an essentially immediate suppression of the axion probability. Moreover, as we shall verify later in this section, this decoherence time is substantially smaller than the lifetime of the heaviest Kaluza-Klein mode contained within $a^{\prime}$. This decoherence mechanism therefore renders the axion superposition $a^{\prime}$ virtually invisible with respect to subsequent axion interactions involving $a^{\prime}$.

It is straightforward to understand this suppression at an intuitive level. Unlike the case with the simple axion zero mode $a_{0}$, in the present case our initial axion state is the infinite linear superposition $a^{\prime}$ given in Eq. (21). Let us therefore consider the behavior of the individual terms in the probability sum (56) as $n_{\max }$ gets large. For this purpose we may drop all factors of two and focus only on the behavior of $P_{a^{\prime} \rightarrow a^{\prime}}$ as a function of $n_{\max }$. For fixed $y$ and large eigenvalues $\tilde{\lambda}$, we have $\tilde{\lambda}^{4} A_{\lambda}^{2} \approx 2$. Therefore, as we introduce increasingly heavy eigenvalues into the probability sum (56), we find the effective behavior 


$$
P_{a^{\prime} \rightarrow a^{\prime}}(t) \sim \frac{1}{n_{\max }^{2}}\left[\sum_{\lambda}(1)+2 \sum_{\lambda^{\prime}<\lambda}(1) \cos \left(\frac{\left[\lambda^{2}-\left(\lambda^{\prime}\right)^{2}\right] t}{2 p}\right)\right]
$$

where each $\lambda$ sum contains $n_{\max }$ terms. For $t=0$, this result factorizes to take the simple leading form

$$
P_{a^{\prime} \rightarrow a^{\prime}}(0) \sim \frac{1}{n_{\max }^{2}} \sum_{\lambda, \lambda^{\prime}}(1+1) \sim \frac{1}{n_{\max }^{2}} n_{\max }^{2} .
$$

In other words, the presence of two independent $\lambda$ sums provides an effective factor of $n_{\max }^{2}$ which cancels the factor of $n_{\max }^{-2}$ that resulted from the normalization of $a^{\prime}$. This initial coherent contribution of the independent two $\lambda$ sums enables the initial probability to start at 1 regardless of the size of the volume of the compactified space. Indeed, the initial state $a^{\prime}$ is a highly coherent state. However, at later times $t>0$, the cosine terms in Eq. (63) no longer add coherently to the sum, and their destructive interference effectively causes the sum to scale only as $n_{\max }$, corresponding to a single diagonal $\lambda$ sum:

$$
P_{a^{\prime} \rightarrow a^{\prime}}(t) \sim \frac{1}{n_{\max }^{2}} \sum_{\lambda}(1) \sim \frac{1}{n_{\max }^{2}} n_{\max } \sim \frac{1}{n_{\max }} .
$$

This phenomenon is completely analogous to the fact that a random walk traverses only the square root of the distance traversed by a coherent, directed walk. It is for this reason that the net axion preservation probability $P_{a^{\prime} \rightarrow a^{\prime}}(t)$ is so strikingly suppressed. Essentially, the initial axion state $a^{\prime}$ has "decohered" as a result of the incoherent Kaluza-Klein oscillations induced by the non-diagonal axion mass matrix.

We thus conclude that in higher dimensions, all axionmediated processes which rely on the production and subsequent detection of the $a^{\prime}$ mode are strongly suppressed by a rapid "decoherence" which renders them virtually "invisible." This decoherence arises as a result of the destructive interference of the infinite-component laboratory axion oscillations. We see, then, that this provides an entirely new higher-dimensional mechanism which can contribute to the invisibility of $a^{\prime}$-mediated processes.

It is important to stress that this decoherence mechanism is relevant only for those processes which are sensitive to the time evolution of the axion. As we have indicated, this includes all axion-mediated process (e.g., axion-exchange processes), as well as processes in which the axion is directly detected in the laboratory. Moreover, measurements of the axion flux from the Sun or from supernovae also fall into this category. However, this does not include processes which are insensitive to axion time evolution. These include, for example, axion-production processes in which the axion appears only as missing energy. Nevertheless, invisibility can be achieved for such processes by adjusting the value of $\hat{f}_{\mathrm{PQ}}$ via the mechanisms discussed in Secs. II and IV.

Finally, we remark that our higher-dimensional decoherence mechanism is completely general, and applies not only to axions, but also to any bulk field $\phi$ whose "shadow" interaction with the standard-model brane involves a coupling between standard-model fields and a "brane shadow" Kaluza-Klein superposition

$$
\phi(y=0)=\sum_{k} c_{k} \phi_{k}
$$

with non-zero coefficients $c_{k}$. Just as in the axion case considered above, the non-trivial time-evolution of the KaluzaKlein modes will cause the initial superposition $\phi(y=0)$ to "decohere" extremely rapidly. This will necessarily produce a severe damping of any standard-model process on the brane that involves couplings to the coherent state $\phi(y$ $=0$ ). For example, this might therefore provide a partial solution to the notorious dilaton problem in string theory: it may simply be that the string-theoretic dilaton is "invisible," in much the same way as the axion is invisible. Similar considerations may also apply to Kaluza-Klein gravitons as well as other bulk moduli fields.

\section{Laboratory oscillations inducing $a_{k} \rightarrow a^{\prime}$}

Finally, let us consider a third relevant oscillation probability. As we discussed above, fields on the standard-model brane can couple only to the linear combination $a^{\prime}$. This is why the probability $P_{a^{\prime} \rightarrow a^{\prime}}(t)$ is the appropriate probability for processes involving both standard-model production and detection of axions. However, for axions that are produced through mechanisms involving bulk fields (which are nonlocalized) rather than brane fields (which are localized), it is possible to envisage situations in which a single KaluzaKlein mode $a_{k}$ (e.g., the zero mode $a_{0}$ ) is produced. However, detection of such an axion mode on our standard-model brane continues to involve couplings to $a^{\prime}$, and therefore in such cases a relevant probability for detection is given by

$$
\begin{aligned}
P_{a_{0} \rightarrow a^{\prime}}(t)= & \frac{1}{N}\left|\sum_{l=0}^{\infty} r_{l} A_{0 \rightarrow l}(t)\right|^{2} \\
= & \frac{1}{N}\left[\sum_{\lambda} \tilde{\lambda}^{4} A_{\lambda}^{4} e^{-\Gamma_{\lambda} t}\right. \\
& +2 \sum_{\lambda^{\prime}<\lambda}{\tilde{\lambda^{2}}}^{2}\left({\tilde{\lambda^{\prime}}}^{\prime} A_{\lambda}^{2} A_{\lambda^{\prime}}^{2} e^{-\left(\Gamma_{\lambda}+\Gamma_{\lambda^{\prime}}\right) t / 2}\right. \\
& \left.\times \cos \left(\frac{\left[\lambda^{2}-\left(\lambda^{\prime}\right)^{2}\right] t}{2 p}\right)\right] .
\end{aligned}
$$

However, just as in the previous case, this probability vanishes in the limit $n_{\max } \rightarrow \infty$, even if we again set $\Gamma_{\lambda}=0$. Specifically, the probability to produce the specific coherent state $a^{\prime}$ on the standard-model brane vanishes as $n_{\max } \rightarrow \infty$. Moreover, the same is true for all probabilities $P_{a_{k} \rightarrow a^{\prime}}(t)$ for all $k$ and for all $y$. Of course, we cannot interpret this result as a decoherence, since even the initial probability at $t=0$ vanishes as $n_{\max } \rightarrow \infty$ due to the negligible overlap between $a_{k}$ and $a^{\prime}$. Nevertheless, this demonstrates that even if the 
axion is produced by bulk fields not restricted to the standard-model brane, the probability for its subsequent detection in the laboratory is vanishingly small.

\section{Axion detection and decay}

Finally, we shall conclude this section by discussing a number of additional effects that arise due to the existence of an infinite tower of excited Kaluza-Klein axion states. Our comments in this subsection will primarily be focused on the possibility of axion detection and in particular on the role of axion decays.

First, as already mentioned above, we must distinguish between processes in which the time-evolution of the axion plays a role and processes which are insensitive to the timeevolution of the axion. For example, the latter include axionproduction processes in which the axion is emitted into the bulk and therefore is manifested on the standard-model brane only as missing energy. A simple example of this is the axion-emission process

$$
F \widetilde{F} \rightarrow a^{\prime} \rightarrow \text { bulk }
$$

where the axion, once produced, flies into the bulk. As we have seen in Sec. II, this process scales as $1 / f_{\mathrm{PQ}}$ rather than $1 / \hat{f}_{\mathrm{PQ}}$. This already leads to the severe constraint $f_{\mathrm{PQ}}$ $\gtrsim \mathcal{O}(\mathrm{TeV})$, and is completely analogous to the possibilities for detecting graviton emission in upcoming $\mathrm{TeV}$-scale collider experiments. We shall discuss one such missing-energy signature in more detail in Sec. V. ${ }^{4}$

By contrast, processes which involve an actual detection of the axion on the standard-model brane are necessarily time-dependent because they involve a non-zero time interval between axion production and axion detection. However, even within this category of time-dependent processes, there are further subdivisions that can be made. One important distinction is the length of the time interval between axion production and axion detection. Certain axion-mediated processes, such as those taking place entirely within accelerator experiments, take place on time scales that are very short compared to the axion lifetime. By contrast, others (such as those involving fluxes of axions which are produced in the Sun or in supernovae and which are subsequently detected on Earth) involve much longer time scales.

\footnotetext{
${ }^{4}$ The rough bound $f_{\mathrm{PQ}} \gtrsim \mathcal{O}(\mathrm{TeV})$ quoted above is primarily a collider bound, but it is natural to wonder whether supernova or redgiant constraints might be more severe. However, the TeV-scale axion scenario envisioned here is safe with respect to these astrophysical constraints for the same reason that the $\mathrm{TeV}$-scale graviton scenarios are safe: the number of Kaluza-Klein modes that are available to carry energy into the bulk depends crucially on the temperature of the relevant astrophysical object, and these temperatures are typically far smaller than the $\mathrm{TeV}$-sized fundamental energy scale of the theory. A detailed discussion of this point in the gravitational context can be found in Ref. [10], and will not be discussed further in this paper.
}

This distinction between "long" and "short" time scales therefore depends on the axion lifetime. Ordinarily, in four dimensions, the axion is relatively long-lived because it is so light and so weakly coupled to ordinary matter. Indeed, the dominant axion decay mode is to two photons, yielding the lifetime

$$
\tau_{4 \mathrm{D}}(a \rightarrow \gamma \gamma)=\frac{1}{\Gamma_{4 \mathrm{D}}(a \rightarrow \gamma \gamma)} \approx\left(\frac{4 \pi}{\alpha}\right)^{2}\left(\frac{\hat{f}_{\mathrm{PQ}}^{2}}{m_{\mathrm{PQ}}^{3}}\right) \approx 10^{48} \mathrm{sec} .
$$

In this expression, we are writing $\hat{f}_{\mathrm{PQ}} \approx 10^{12} \mathrm{GeV}$ to denote the usual four-dimensional Peccei-Quinn symmetry-breaking scale, and taking $m_{\mathrm{PQ}} \approx 10^{-5} \mathrm{eV}$ to denote the usual fourdimensional axion mass. (We choose these symbols in order to facilitate the comparison between the four-dimensional and higher-dimensional situations.) We are also disregarding numerous model-dependent $\mathcal{O}(1)$ coefficients which do not affect the overall scale of the result. Thus, in four dimensions, the axion is extraordinarily stable.

In higher dimensions, this situation changes dramatically. Of course, the coherent axion mode $a^{\prime}$ is not a mass eigenstate, and thus, strictly speaking, it does not have a welldefined "lifetime." Nevertheless, we can determine an effective lifetime for $a^{\prime}$ by estimating the shortest lifetime of any of the mass eigenstates $\hat{a}_{\lambda}$ of which it is comprised. This then yields the time scale over which the coherent state $a^{\prime}$ naturally decays as a result of its couplings to ordinary matter. In general, it is the heaviest Kaluza-Klein mass eigenstates which have the shortest lifetimes, with the decay mode into two photons continuing to be dominant. As a function of the cutoff $n_{\max }$, the "lifetime" of the coherent state $a^{\prime}$ can therefore be estimated to be

$$
\begin{aligned}
\tau_{D>4}\left(a^{\prime} \rightarrow \gamma \gamma\right) & =\frac{1}{\Gamma_{4 \mathrm{D}}\left(\hat{a}_{\lambda_{\max }} \rightarrow \gamma \gamma\right)} \\
& \approx\left(\frac{4 \pi}{\alpha}\right)^{2}\left(\frac{\hat{f}_{\mathrm{PQ}}^{2}}{M_{\text {string }}^{3}}\right) \\
& =\left(\frac{m_{\mathrm{PQ}}}{M_{\text {string }}}\right)^{3} \tau_{4 \mathrm{D}}(a \rightarrow \gamma \gamma) \\
& \approx 10^{-3} \mathrm{sec} .
\end{aligned}
$$

In this expression we have taken $n_{\text {max }} \approx R M_{\text {string }}$, implying $\lambda_{\max } \approx M_{\text {string }}$. We have also chosen $M_{\text {string }}=1 \mathrm{TeV}$ for simplicity. Thus, we see that the coherent state can be expected to decay to two photons much more rapidly than the usual four-dimensional axion. Of course, in this calculation we have taken the cutoff $\lambda_{\max } \approx M_{\text {string }}$, which represents the "worst-case" scenario. In any axion-related process of total energy $E<M_{\text {string }}$, the production of Kaluza-Klein axion modes $\hat{a}_{\lambda}$ with $\lambda \gtrsim E$ will be kinematically disfavored. We would then take $\lambda_{\max } \approx E$, which can increase the lifetime considerably.

A priori, this significant reduction in the axion lifetime relative to the four-dimensional case means that our "deco- 
herence" phenomenon for $a^{\prime}$ is irrelevant unless the decoherence time $\tau_{0}$ is even smaller. However, it is straightforward to verify that this is indeed the case. Consulting Eq. (61), we see that

$$
\tau_{0} \approx y^{4}\left(\frac{p}{1 \mathrm{TeV}}\right)\left(\frac{M_{\text {string }}}{1 \mathrm{TeV}}\right)^{-2} \times 10^{-33} \mathrm{sec}
$$

where we have taken $n_{\max }^{\prime} \approx R M_{\text {string }}$. For all phenomenologically interesting values of $p$ and $M_{\text {string }}$, we thus conclude that $\tau_{0} \ll \tau_{D>4}$. We see, therefore, that the coherent axion mode $a^{\prime}$ indeed decoheres sufficiently rapidly to justify the $\Gamma_{\lambda}=0$ approximation that was used in deriving it. ${ }^{5}$

Given that the coherent state $a^{\prime}$ has an intrinsic lifetime $\tau_{a^{\prime}} \approx 10^{-3} \mathrm{sec}$, we can therefore use this as a benchmark for separating "long" and "short" processes. For laboratory oscillations over time scales shorter than this, it is legitimate to neglect the axion decay widths in calculations of these oscillations. By contrast, calculations of oscillations over longer time scales require the inclusion of the decay widths, and will therefore start to feel the effects of axion decays.

It is therefore important to understand the effects of such axion decays, particularly as they relate to axion "invisibility." For this purpose, we may draw another distinction, this time between processes that take place entirely within a single detector (such as an axion-mediated process $F \widetilde{F} \rightarrow a^{\prime}$ $\rightarrow F \widetilde{F}$ ), and those which take place largely outside our detector (such as an axion beam travelling from the Sun to the Earth). For processes taking place entirely within a single detector, axion decay represents a breakdown of invisibility because we can in principle detect the emitted decay products. For example, even though we have found that $P_{a^{\prime} \rightarrow a^{\prime}}(t)$ is suppressed for all times $t$ exceeding the decoherence time $\tau_{0}$, the decohered state will nevertheless continue to propagate until the individual Kaluza-Klein axion modes that comprise this decohered state themselves decay. This will be discussed in more detail below. Given this observation, one might initially doubt the phenomenological importance of the $a^{\prime}$ decoherence phenomenon. However, the important point is that it is only the coherent state $a^{\prime}$ which couples to standard-model fields with the potentially dangerous unsuppressed coupling $1 / f_{\mathrm{PQ}}$, whereas individual Kaluza-Klein axion modes instead experience the safer suppressed coupling $1 / \hat{f}_{\mathrm{PQ}}$. The decoherence phenomenon therefore indicates that there is only a vanishingly small time interval during which a process of the form $F \widetilde{F} \rightarrow a^{\prime} \rightarrow F \widetilde{F}$ can possibly occur with a dangerously large amplitude scaling as $1 / f_{\mathrm{PQ}}^{2}$. After this initial time interval, the $a^{\prime}$ state

\footnotetext{
${ }^{5}$ In comparing Eqs. (70) and (71), we must actually account for the relative rescaling of the cutoff $n_{\max } \rightarrow n_{\max }^{\prime}$, as discussed above Eq. (61). This introduces an additional multiplicative factor $\left(y^{2} / 981\right)^{3}$ into Eq. (70), thereby shortening $\tau_{D>4}$ by an additional factor $\approx 10^{-9}$ for $y \approx \mathcal{O}(1)$. However, we see from Eq. (71) that this still does not affect our main conclusion that $\tau_{0} \ll \tau_{D>4}$.
}

decoheres, and all subsequent interactions and decays will have amplitudes scaling as $1 / \hat{f}_{\mathrm{PQ}}^{2}$.

In sharp contrast are processes which largely take place outside our detectors. In such cases, we do not expect to be able to detect the decay products that result from axion decays at intermediate times. For example, in the case of an axion beam travelling from the Sun to the Earth, the photons emitted in flight through axion decays are presumably indistinguishable from the general background radiation. In such situations, therefore, axion decays lead not to a loss of invisibility, but rather to an enhancement of it. For example, let us consider the probability that the initial state $a^{\prime}$ will be found in a particular mass eigenstate $\hat{a}_{\lambda}$ as function of time. Following the same procedure as above and using Eq. (45), we find the probability

$$
P_{a^{\prime} \rightarrow \hat{a}_{\lambda}}(t)=\frac{1}{N} \tilde{\lambda}^{4} A_{\lambda}^{2} e^{-\Gamma_{\lambda} t}
$$

Note that this is an exact result valid for all times, with axion decays producing an exponential suppression for the probability. Moreover, as we shall now demonstrate, this fact can be used to provide a direct experimental test of the higherdimensional nature of the axion. Using Eq. (72), we may define

$$
P_{\mathrm{tot}}(t) \equiv \sum_{\lambda} P_{a^{\prime} \rightarrow \hat{a}_{\lambda}}=\frac{1}{N} \sum_{\lambda} \tilde{\lambda}^{4} A_{\lambda}^{2} e^{-\Gamma_{\lambda} t}
$$

as a "collective" amplitude that the axion mode $a^{\prime}$ survives as a function of time. This interpretation is justified because $P_{\text {tot }}(t)$ is nothing but the time-dependent norm of the original $a^{\prime}$ superposition:

$$
P_{\mathrm{tot}}(t)=\left\langle a^{\prime}(t) \mid a^{\prime}(t)\right\rangle
$$

Given this norm, the collective decay width (i.e., the instantaneous decay probability per unit time) is given as

$$
\langle\Gamma\rangle \equiv-\frac{1}{P_{\text {tot }}} \frac{d P_{\text {tot }}(t)}{d t}=\frac{\sum_{\lambda} \Gamma_{\lambda} \tilde{\lambda}^{4} A_{\lambda}^{2} e^{-\Gamma_{\lambda} t}}{\sum_{\lambda} \tilde{\lambda}^{4} A_{\lambda}^{2} e^{-\Gamma_{\lambda} t}},
$$

in complete analogy with the formalism for radioactive decays. However, because of the presence of an infinite tower of Kaluza-Klein states, it is immediately apparent that $\langle\Gamma\rangle$ is itself a function of time. For example, at very early times we have $\langle\Gamma\rangle \sim \Gamma_{\lambda_{\max }} \sim M_{\text {string }}^{3} / \hat{f}_{\mathrm{PQ}}^{2}$, where $\lambda_{\text {max }} \approx M_{\text {string }}$ is the heaviest mass eigenvalue included in the linear superposition. By contrast, at extremely late times, we have $\langle\Gamma\rangle$ $\rightarrow \Gamma_{\lambda_{0}}$ where $\lambda_{0}$ is the lightest mass eigenvalue. Since $\langle\Gamma\rangle$ is the instantaneous decay probability per unit time (which can be measured in our Earth-bound detector), a time-variation in $\langle\Gamma\rangle$ from different axion sources at different distances would 
serve as a direct experimental test of the higher-dimensional nature of the axion. ${ }^{6}$

Finally, let us briefly comment on the possibility of measuring the laboratory axion oscillations discussed previously. Although we have shown that individual probabilities such as $P_{a_{k} \rightarrow a_{l}}(t)$ experience sinusoidal oscillations as functions of time, at a physical level these calculations presuppose that we are capable of experimentally detecting individual Kaluza-Klein modes $a_{k}$. In other words, these calculations presuppose that we are able to distinguish the final axion states according to their Kaluza-Klein quantum numbers. In situations where such detections can be made, our previous results continue to apply. However, in the most straightforward scenarios, all Kaluza-Klein states will have identical decay modes (e.g., into two photons), leaving us with no experimental "handle" through which to detect the presence of an individual oscillation. Thus, the prospects for detecting individual laboratory axion oscillations depend crucially on the ability to perform laboratory axion measurements which are sensitive to particular Kaluza-Klein quantum numbers. Of course, this is completely analogous to the case of neutrino oscillations, where the existence of neutrino decay modes that distinguish between different neutrino quantum numbers [such as $S U(2)$ gauge charge or flavor] permits the detection of neutrino oscillations. While it is not hard to imagine scenarios in which Kaluza-Klein selection rules might be exploited in order to make such individual measurements in the axion case, the details of such scenarios are expected to be highly model-dependent since they necessarily depend on the specific geometry of the compactification. We shall therefore not consider this issue further. Regardless of this issue, however, the most important experimental signature of these oscillations is the net decoherence that they induce for the linear superposition $a^{\prime}$. As we have seen, this decoherence is striking and renders the axion invisible with respect to subsequent laboratory interactions.

\section{COSMOLOGICAL RELIC AXION OSCILLATIONS}

In this section, we shall discuss a third higherdimensional effect that can contribute to the "invisibility" of axions: the rate at which the energy trapped in cosmological relic axion oscillations is dissipated. As we shall see, the presence of an infinite tower of Kaluza-Klein axion states can, under certain circumstances, actually enhance the rate at which this oscillation energy is dissipated. In such cases, the effective Peccei-Quinn scale $\hat{f}_{\mathrm{PQ}}$ can therefore be raised beyond its usual relic-oscillation bounds, leading to an axion

\footnotetext{
${ }^{6}$ After this paper originally appeared, some of these ideas concerning the phenomenological consequences of solar Kaluza-Klein axions were further investigated in Ref. [21]. It was found that heavy Kaluza-Klein modes can be measured experimentally with a feasible terrestrial detector without significant background interference. It was also shown that the decays of solar Kaluza-Klein axions do not produce unacceptable amounts of x-ray luminosity. This is therefore an interesting experimental possibility for detecting solar Kaluza-Klein axions.
}

whose couplings to matter are even more suppressed than in four dimensions. This can therefore provide another higherdimensional way of achieving an "invisible" axion.

Let us first recall the situation in four dimensions. One of the most important constraints on the scale of Peccei-Quinn symmetry breaking, and hence on the mass of the axion and the strength of its couplings to ordinary matter, comes from cosmological relic axion oscillations. Unlike the laboratory oscillations discussed in the previous section, these cosmological relic oscillations arise due to the fact that as the universe cools and passes through the QCD phase transition at $T \approx \Lambda_{\mathrm{QCD}}$, instanton effects suddenly establish a non-zero axion potential where none previously existed. In the usual four-dimensional situation, the axion can therefore find itself displaced relative to the newly-established minimum of the potential, and begin to oscillate around it according to the differential equation

$$
\frac{d^{2} a}{d t^{2}}+3 H(t) \frac{d a}{d t}+m_{a}^{2} a=0, \quad t \gtrsim t_{\mathrm{QCD}} .
$$

Here $H(t)=1 /(2 t)$ is the Hubble constant, where we are assuming for simplicity that this time-evolution takes place entirely in a radiation-dominated universe. We are also assuming that the axion mass $m_{a}$ is independent of time for $t$ $\geqslant t_{\mathrm{QCD}}$ and vanishes for times $t \leqslant t_{\mathrm{QCD}}$. We are also neglecting the decay width of the axion. In general, the initial amplitude of this oscillation at $t_{\mathrm{QCD}}$ is set by the initial random angular displacement of the axion field. This scales as $f_{\mathrm{PQ}}$, where $f_{\mathrm{PQ}}$ is the axion decay constant. Although these oscillations are ultimately damped due the cosmological Hubble expansion term, a relic of these axion oscillations should still exist today. Imposing the requirement that the energy stored in the relic oscillation today be less than the critical energy density (so as not to overclose the universe) then sets an upper bound $f_{\mathrm{PQ}} \leq 10^{12} \mathrm{GeV}$ which is consistent with bounds obtained through other means. This is therefore a bound on the "invisibility" of the axion.

At first glance, it might seem that this issue should no longer play a role in our higher-dimensional scenario in which the fundamental scale $f_{\mathrm{PQ}}$ of Peccei-Quinn symmetry breaking is substantially lowered (perhaps even to the TeVrange) as a result of the volume factor in Eq. (19). Indeed, it is the (low) fundamental mass scale $f_{\mathrm{PQ}}$ rather than the (high) effective mass scale $\hat{f}_{\mathrm{PQ}}$ which sets the size of the vacuum expectation value (VEV) of the axion field $a$ in the five-dimensional Lagrangian (14), and which similarly sets the overall scale of the initial random angular displacement of the axion field. However, in passing from Eq. (14) to the four-dimensional Lagrangian (17), there is an implicit volume-dependent rescaling of the axion field, as discussed below Eq. (18). Thus, even though initial displacement of the unrescaled axion field scales with $f_{\mathrm{PQ}}$, the initial displacement of the rescaled axion field scales with $\hat{f}_{\mathrm{PQ}}$. The danger of an excessive oscillation energy density today is therefore just as relevant for our higher-dimensional scenario as it is for the usual four-dimensional case. 
In fact, this danger may actually be greater in the higherdimensional case. This is because the Kaluza-Klein reduction yields not only the usual zero-mode axion $a_{0}$, but also an infinite tower of excited Kaluza-Klein axions $a_{k}$ ( $k$ $>0)$. The above differential equation for the axion oscillations then generalizes to

$$
\frac{d^{2} a_{k}}{d t^{2}}+3 H(t) \frac{d a_{k}}{d t}+\mathcal{M}_{k l}^{2} a_{l}=0, \quad t \gtrsim t_{\mathrm{QCD}}
$$

where $\mathcal{M}^{2}$ is the non-diagonal mass matrix given in Eq. (35). (Note that we shall continue to neglect axion decay widths in these equations; this assumption will be justified at the end of this section.) Of course, due to their Kaluza-Klein masses, these excited Kaluza-Klein axions feel a non-zero potential even prior to the QCD phase transition (i.e., even prior to the "turn-on" of $m_{\mathrm{PQ}}$ ), and it is therefore reasonable to assume that they are already sitting at their minima at the time of the QCD phase transition. However, due to the nondiagonal mass mixing matrix in Eq. (77), the initial displacement of only the zero-mode axion $a_{0}$ is sufficient to trigger the excited Kaluza-Klein modes into oscillation. This situation is illustrated in Fig. 4.

Given this observation, there are a priori three possible effects that these excited Kaluza-Klein states can have on the system. First, it is possible that these excited Kaluza-Klein states will "capture" oscillation energy from the zero-mode oscillation, and essentially store it. Thus, in this case, the total energy density of the system would dissipate more slowly, thereby resulting in a greater relic energy density today. This would seriously strengthen the five-dimensional axion bounds relative to the usual four-dimensional bounds, and provide the most serious threat to the viability of these higher-dimensional scenarios. The second possibility is that although the excited Kaluza-Klein states steal energy from the zero-mode oscillation, they may be able to dissipate it more effectively. This would then lead to an enhanced relic energy loss rate, implying a weakened bound on the higherdimensional scenarios. Finally, the third possibility is that the two effects cancel exactly, with the excited Kaluza-Klein states oscillating in such a way that even though they capture some energy from the zero mode, they also alter the timedevelopment of the zero mode in an exactly compensatory manner. Thus the traditional four-dimensional bounds would remain unchanged.

In order to determine which of these possibilities is realized, we must solve the coupled differential equations (77). In so doing, we shall make the following assumptions. First, we shall assume that prior to the QCD phase transition at $t_{\mathrm{QCD}}$, the axion zero-mode experiences no potential and therefore has an initial displacement set to 1 (in units of $\left.\hat{f}_{\mathrm{PQ}}\right)$. We shall likewise assume that $d a_{0} / d t=0$ at $t$ $=t_{\mathrm{QCD}}$. By contrast, for the excited Kaluza-Klein modes $a_{k}(k>0)$, we shall begin with the initial conditions $a_{k}$ $=d a_{k} / d t=0$ at $t_{\mathrm{QCD}}$. These conditions reflect the fact that these excited modes have non-zero Kaluza-Klein masses even prior to the "turn-on" of $m_{\mathrm{PQ}}$ at $t_{\mathrm{QCD}}$, and hence should have essentially settled into their minima prior to the QCD phase transition. Although it is possible for the lighter

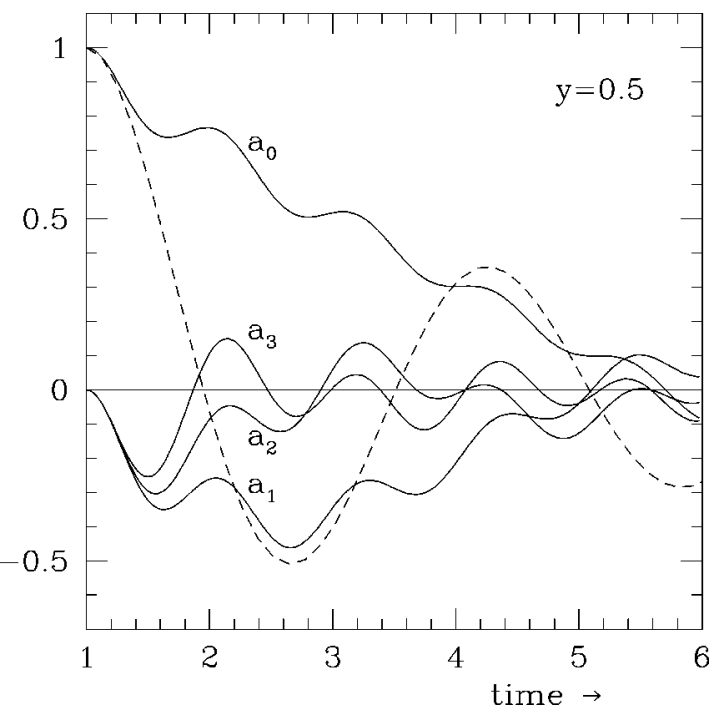

FIG. 4. A plot of the coupled Kaluza-Klein cosmological relic axion oscillations. For this plot, we have normalized the initial displacement of the axion zero mode to 1 , and taken $y=0.5, m_{\mathrm{PQ}}$ $=2$, and $t_{\mathrm{QCD}}=1$ (in dimensionless units). We have also considered the effects of only the first three excited Kaluza-Klein modes. Although the excited Kaluza-Klein modes have vanishing initial displacements, they are triggered into oscillation as a result of the initial displacement of the zero mode. This in turn changes the subsequent time evolution of the zero mode. By contrast, the superimposed dashed line shows the behavior of the usual fourdimensional axion zero mode in the $y \rightarrow \infty$ limit (i.e., the usual four-dimensional case when no Kaluza-Klein modes are present).

Kaluza-Klein axion modes to have small displacements as well, for simplicity we shall ignore this possibility in what follows. Note that this assumption also enables us to start with identical initial relic oscillation energy densities in both the four- and five-dimensional situations, and thereby enables us to make a direct comparison of the effects of the Kaluza-Klein modes on the cosmological time-evolution of the system. Finally, in substituting the matrix (35) into Eq. (77), we shall assume that $m_{\mathrm{PQ}}(t)$ is given exactly by the step function

$$
m_{\mathrm{PQ}}(t)=m_{\mathrm{PQ}} \Theta\left(t-t_{\mathrm{QCD}}\right)
$$

The constancy of $m_{\mathrm{PQ}}(t)$ for $t>t_{\mathrm{QCD}}$ is often referred to as the "adiabatic'" approximation. We caution, however, that for large values of $m_{\mathrm{PQ}}$ this step-function approximation can differ quite substantially from the results of a more careful analysis of the time-temperature dependence of the axion mass due to instanton effects [19].

Given these assumptions, it turns out to be possible to solve the differential equations (77) analytically for all times $t \geqslant t_{\mathrm{QCD}}$ and for an arbitrary number of Kaluza-Klein modes. We therefore do not need to make any further approximations (such as the traditional separation into so-called "overdamped," "underdamped,' and "critically damped" oscillation phases). The first step in our analytical solution is to decouple the differential equations (77) by passing to the mass-eigenstate basis $\hat{a}_{\lambda}$ defined in Eq. (39). In order to 
work with dimensionless quantities, we shall define $\tilde{a}_{\lambda}$ $\equiv \hat{a}_{\lambda} / \hat{f}_{\mathrm{PQ}}$. Each of our uncoupled differential equations then takes the form

$$
\frac{d^{2} \tilde{a}_{\lambda}}{d t^{2}}+\frac{3}{2 t} \frac{d \tilde{a}_{\lambda}}{d t}+\lambda^{2} \tilde{a}_{\lambda}=0
$$

For simplicity we can recast this equation into the form

$$
\frac{d^{2} \tilde{a}_{\lambda}}{d \tau^{2}}+\frac{3}{2 \tau} \frac{d \tilde{a}_{\lambda}}{d \tau}+\tilde{a}_{\lambda}=0
$$

where we have defined $\tau \equiv \lambda t$. The most general solution to this equation is then given by

$$
\tilde{a}(\tau)=\tau^{-1 / 4}\left[c_{+} J_{1 / 4}(\tau)+c_{-} J_{-1 / 4}(\tau)\right]
$$

where $J_{\nu}(\tau)$ are the Bessel functions of first kind. We therefore wish to solve for the unknown constant coefficients $c_{+}$ and $c_{-}$. To do this, we impose our initial conditions. In the original Kaluza-Klein basis, these conditions are given by $a_{k}\left(\tau_{0}\right)=\hat{f}_{\mathrm{PQ}} \delta_{k 0}$ and $d a_{k}\left(\tau_{0}\right) / d \tau=0$ where $\tau_{0} \equiv \tau_{\mathrm{QCD}}$ is the initial time at which we begin the time evolution of our axion fields (representing the "turn-on" time for $m_{\mathrm{PQ}}$ ). In the dimensionless mass-eigenstate basis, these initial conditions therefore take the form:

$$
\tilde{a}_{\lambda}\left(\tau_{0}\right)=A_{\lambda},\left.\quad \frac{d \tilde{a}_{\lambda}}{d \tau}\right|_{\tau=\tau_{0}}=0
$$

where $A_{\lambda}$ is defined in Eq. (41). The assumptions underlying these initial conditions were discussed above. Thus, in the mass-eigenstate basis, we see that each of the Kaluza-Klein modes begins with an initial displacement. Solving for $c_{ \pm}$is then straightforward. The first initial condition (the displacement condition) trivially gives the constraint

$$
c_{+} J_{1 / 4}+c_{-} J_{-1 / 4}=A_{\lambda} \tau_{0}^{1 / 4}
$$

where for notational convenience any Bessel function written without an argument is understood to be evaluated at $\tau_{0}$. Using the Bessel-function identity

$$
\frac{d}{d \tau}\left[\tau^{ \pm \nu} J_{\nu}(\tau)\right]= \pm \tau^{ \pm \nu} J_{\nu \mp 1}(\tau),
$$

we see that the initial velocity constraint takes the form

$$
\frac{d \tilde{a}_{\lambda}}{d \tau}=\tau^{-1 / 4}\left[-c_{+} J_{5 / 4}(\tau)+c_{-} J_{-5 / 4}(\tau)\right],
$$

implying $c_{+} J_{5 / 4}=c_{-} J_{-5 / 4}$. Together with Eq. (83), this leads to the solutions

$$
c_{ \pm}=-\frac{\pi}{\sqrt{2}} A_{\lambda} \tau_{0}^{5 / 4} J_{\mp 5 / 4}
$$

where we have used the further identity

$$
J_{1 / 4} J_{-5 / 4}+J_{-1 / 4} J_{5 / 4}=-\frac{\sqrt{2}}{\pi \tau_{0}} .
$$

Substituting $c_{ \pm}$from Eq. (86) into the solution given in Eq. (81), we thus obtain our final closed-form solution to the axion differential equation:

$$
\tilde{a}_{\lambda}(\tau)=-\frac{\pi}{\sqrt{2}} A_{\lambda} \tau_{0}^{5 / 4} \tau^{-1 / 4} j\left(\tau_{0} ; \tau\right)
$$

where we have defined

$$
j\left(\tau_{0} ; \tau\right) \equiv J_{-5 / 4}\left(\tau_{0}\right) J_{1 / 4}(\tau)+J_{5 / 4}\left(\tau_{0}\right) J_{-1 / 4}(\tau) .
$$

This implies that the first time derivative is given by

$$
\frac{d \tilde{a}_{\lambda}}{d \tau}=\frac{\pi}{\sqrt{2}} A_{\lambda} \tau_{0}^{5 / 4} \tau^{-1 / 4} j^{\prime}\left(\tau_{0} ; \tau\right)
$$

where we have likewise defined

$$
j^{\prime}\left(\tau_{0} ; \tau\right) \equiv J_{-5 / 4}\left(\tau_{0}\right) J_{5 / 4}(\tau)-J_{5 / 4}\left(\tau_{0}\right) J_{-5 / 4}(\tau) .
$$

Note that $j^{\prime}\left(\tau_{0} ; \tau\right) \rightarrow 0$ as $\tau \rightarrow \tau_{0}$, as expected, since the initial velocities vanish for each of the axion modes.

Now, the energy contribution from a single mode $\tilde{a}_{\lambda}$ is given by

$$
\tilde{\rho}_{\lambda}(\tau) \equiv \frac{\tilde{\lambda}^{2}}{2}\left[\tilde{a}_{\lambda}^{2}+\left(\frac{d \tilde{a}_{\lambda}}{d \tau}\right)^{2}\right]
$$

where $\tilde{\lambda}$ is the dimensionless eigenvalue defined in Eq. (37) and where $\tilde{\rho} \equiv \rho /\left(m_{\mathrm{PQ}}^{2} \hat{f}_{\mathrm{PQ}}^{2}\right)$ is a dimensionless energy density. Substituting in the above results, we therefore find

$$
\tilde{\rho}_{\lambda}(\tau)=\frac{\pi^{2}}{4} A_{\lambda}^{2} \widetilde{\lambda}^{2} \tau_{0}^{5 / 2} \tau^{-1 / 2}\left[j\left(\tau_{0} ; \tau\right)^{2}+j^{\prime}\left(\tau_{0} ; \tau\right)^{2}\right]
$$

While this expression is exact for all times $\tau$, it is also useful to have an approximation valid for extremely late times satisfying $\tau \gg 1$ (such as the present cosmological time). Using the Bessel-function asymptotic expansion

$$
J_{\nu}(\tau) \approx \sqrt{\frac{2}{\pi \tau}} \cos \left(\tau-\frac{\pi \nu}{2}-\frac{\pi}{4}\right) \text { as } \tau \rightarrow \infty,
$$

it is straightforward to show that

$$
\begin{aligned}
j\left(\tau_{0} ; \tau\right)^{2}+j^{\prime}\left(\tau_{0} ; \tau\right)^{2} \approx & \frac{2}{\pi \tau}\left\{\left[J_{5 / 4}\left(\tau_{0}\right)\right]^{2}+\left[J_{-5 / 4}\left(\tau_{0}\right)\right]^{2}\right. \\
& \left.+\sqrt{2} J_{5 / 4}\left(\tau_{0}\right) J_{-5 / 4}\left(\tau_{0}\right)\right\} .
\end{aligned}
$$

Given the exact result (93), we can convert from $\tau$ back to our original time variable $t$ to obtain the final closed-form solution 


$$
\tilde{\rho}_{\lambda}(\widetilde{t})=\frac{\pi^{2}}{4} A_{\lambda}^{2} \widetilde{\lambda}^{4} \tilde{t}_{0}^{5 / 2} \widetilde{t}^{-1 / 2}\left[j\left(\tilde{\lambda} \tilde{t}_{0} ; \tilde{\lambda} \widetilde{t}\right)^{2}+j^{\prime}\left(\tilde{\lambda} \widetilde{t}_{0} ; \tilde{\lambda} \widetilde{t}\right)^{2}\right]
$$

where we have defined the dimensionless time $\tilde{t} \equiv m_{\mathrm{PQ}} t$. Thus, adding together the contributions from all the masseigenstate modes, we obtain the final energy density:

$$
\begin{aligned}
\tilde{\rho}(\tilde{t}) & =\sum_{\lambda} \tilde{\rho}_{\lambda}(\tilde{t}) \\
& =\frac{\pi^{2}}{4} \tilde{t}_{0}^{5 / 2} \tilde{t}^{-1 / 2} \sum_{\lambda} A_{\lambda}^{2} \widetilde{\lambda}^{4}\left[j\left(\tilde{\lambda} \tilde{t}_{0} ; \tilde{\lambda} \widetilde{t}\right)^{2}+j^{\prime}\left(\tilde{\lambda} \tilde{t}_{0} ; \tilde{\lambda} \widetilde{t}\right)^{2}\right] .
\end{aligned}
$$

Note that this is the exact result for the relic oscillation energy density as a function of time.

It is easy to verify that $\tilde{\rho}(\tilde{t})$ has the correct limit as $\tilde{t}$ $\rightarrow \widetilde{t}_{0}$. Indeed, as $\widetilde{t} \rightarrow \widetilde{t}_{0}$, we find using the identity (87) that $\tilde{\rho}(t) \rightarrow \tilde{\rho}_{0}$, where

$$
\tilde{\rho}_{0} \equiv \frac{1}{2} \sum_{\lambda} \tilde{\lambda}^{2} A_{\lambda}^{2}
$$

This is indeed the correct value of the initial energy in the mass-eigenstate basis, since each mass eigenmode starts with zero velocity and with initial displacement $A_{\lambda}$. However, in our original Kaluza-Klein basis, our initial conditions at $\tilde{t}_{0}$ consist of having only the zero mode displaced by 1 . This implies that $\tilde{\rho}=1 / 2$. Comparing this result with Eq. (98) then yields the identity quoted in Eq. (43). In particular, this identity holds for all $y$, as can be verified directly by substituting the values of $\lambda$ and $A_{\lambda}$ and evaluating the eigenvalue sum.

For late times $\tilde{\lambda} \tilde{t} \gg 1$, the exact result (97) for the energy density simplifies to take the form

$$
\tilde{\rho}(\tilde{t})=\frac{\pi}{2} X\left(\tilde{t}_{0}\right) \tilde{t}_{0}^{5 / 2} \tilde{t}^{-3 / 2}
$$

where the time-independent coefficient $X\left(\tilde{t}_{0}\right)$ is given by

$$
\begin{aligned}
X\left(\tilde{t}_{0}\right) \equiv & \sum_{\lambda} A_{\lambda}^{2} \widetilde{\lambda}^{3}\left\{\left[J_{5 / 4}\left(\tilde{\lambda} \tilde{t}_{0}\right)\right]^{2}+\left[J_{-5 / 4}\left(\tilde{\lambda} \tilde{t}_{0}\right)\right]^{2}\right. \\
& \left.+\sqrt{2} J_{5 / 4}\left(\tilde{\lambda} \tilde{t}_{0}\right) J_{-5 / 4}\left(\tilde{\lambda} \tilde{t}_{0}\right)\right\} .
\end{aligned}
$$

This demonstrates that asymptotically, the total energy density falls as $\tilde{\rho}(\tilde{t}) \sim \tilde{t}^{-3 / 2}$ regardless of the presence of the excited axion Kaluza-Klein modes. Moreover, in the "double-asymptotic" cases in which we also have $\tilde{\lambda} \widetilde{t}_{0} \gg 1$ for all $\tilde{\lambda}$, we can further approximate

$$
X\left(\tilde{t}_{0}\right) \approx \frac{1}{\pi \tilde{t}_{0}} \sum_{\lambda} \tilde{\lambda}^{2} A_{\lambda}^{2}=\frac{1}{\pi \tilde{t}_{0}},
$$

where we have used the identity (43) in the last equality. We then find

$$
\tilde{\rho}(\tilde{t}) \approx \frac{1}{2}\left(\frac{\tilde{t}}{\tilde{t}_{0}}\right)^{-3 / 2},
$$

which is consistent with the initial energy density $\tilde{\rho}\left(\tilde{t}_{0}\right)$ $=1 / 2$. However, for practical purposes, we shall focus on the expressions (99) and (100) in which $\tilde{t}$ is taken large but $\tilde{t}_{0}$ is kept arbitrary. This is because $\tilde{\lambda} \widetilde{t}_{0}$ can occasionally be relatively small (particularly for the lightest eigenvalues) even if $\tilde{t}_{0}$ itself is relatively large. Finally, in the four-dimensional $y \rightarrow \infty$ limit, we find that $\widetilde{\lambda_{k}} \rightarrow k y$ for $k \geqslant 1$, while $\widetilde{\lambda_{0}} \rightarrow 1$. Thus, in this limit, the excited Kaluza-Klein modes decouple and we obtain the four-dimensional result

$$
X_{4 \mathrm{D}}\left(\tilde{t}_{0}\right)=\left[J_{5 / 4}\left(\tilde{t}_{0}\right)\right]^{2}+\left[J_{-5 / 4}\left(\tilde{t}_{0}\right)\right]^{2}+\sqrt{2} J_{5 / 4}\left(\tilde{t}_{0}\right) J_{-5 / 4}\left(\tilde{t}_{0}\right) .
$$

As expected, this approaches the value given in Eq. (101) for $\tilde{t}_{0} \gg 1$.

We see from Eq. (99) that although the rate of energy loss remains fixed at asymptotic times, the presence of the Kaluza-Klein states can nevertheless change the overall value of the energy. This is because the oscillating excited Kaluza-Klein states can change the rate of energy loss at intermediate times, possibly leading to an enhanced or diminished energy at late times. The cumulative effect of these Kaluza-Klein states at late times is encoded within the expression $X\left(\tilde{t}_{0}\right)$. Therefore, in order to understand the effect of the Kaluza-Klein states on the energy dissipation rate, we need to understand the behavior of $X\left(\widetilde{t}_{0}\right)$ as a function of the radius variable $y \equiv\left(m_{\mathrm{PQ}} R\right)^{-1}$ for a fixed initial time $\tilde{t}_{0}$.

The results are rather surprising. Of course, for $\widetilde{t}_{0} \gg 1$, we are in the "double-asymptotic" regime $\left(\widetilde{t}_{0}, \widetilde{t}\right) \gg 1$ for which Eqs. (101) and (102) are expected to apply. We therefore find that in such cases the presence of the Kaluza-Klein modes does not alter the energy density relative to the energy density that would have been obtained in four dimensions. In other words, even though we have an infinite set of KaluzaKlein axion modes which are induced into oscillation as a result of the initial displacement of the axion zero mode, these oscillations nevertheless change the time-development of the zero mode in a compensatory manner so that the total oscillation energy density as a function of time is exactly preserved. This indicates that in such situations, the higherdimensional axion scenarios are no less viable than the usual four-dimensional scenarios.

Even more surprising, however, is the situation that arises for smaller $\tilde{t}_{0}$. In such cases, we cannot use the "doubleasymptotic" expression (101), and we must resort to the full expression in Eq. (100). We then find that $X\left(\tilde{t}_{0}\right)$ is smaller than the four-dimensional value given in Eq. (103), which implies that

$$
\rho(t)<\rho_{4 \mathrm{D}}(t) .
$$

In other words, even though the excited Kaluza-Klein states are triggered into oscillation by the initial displacement of 


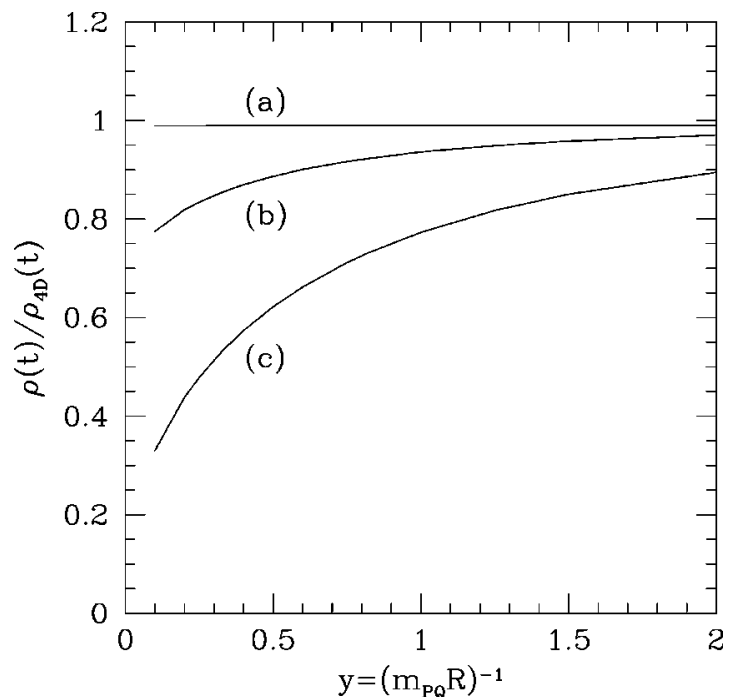

FIG. 5. The energy-dissipation ratio factor $\rho(t) / \rho_{4 \mathrm{D}}(t)$ $=X\left(\tilde{t}_{0}\right) / X_{4 \mathrm{D}}\left(\tilde{t}_{0}\right)$ as a function of $y \equiv\left(m_{\mathrm{PQ}} R\right)^{-1}$, assuming (a) $\tilde{t}_{0}$ $=10$; (b) $\tilde{t}_{0}=1$; and (c) $\tilde{t}_{0}=0.1$. All cases reduce to the usual four-dimensional result as $y \rightarrow \infty$, with the Kaluza-Klein axion states decoupling. However, as the size of the extra dimension grows and $y$ decreases from infinity, we see that the net effect of the Kaluza-Klein states is to dissipate the relic oscillation energy density more rapidly, leading to smaller relic oscillation energy densities at final times. The size of this effect depends on $\tilde{t}_{0}$, with smaller values of $\tilde{t}_{0}$ corresponding to sizable effects at large values of $y$, while for larger values of $\tilde{t}_{0}$ this effect is delayed until correspondingly smaller values of $y$. In general, this effect becomes substantial for $y \tilde{t}_{0} \lesssim \mathcal{O}(1)$. Note that all curves tend to zero in the $y$ $\rightarrow 0$ limit, implying an infinitely rapid dissipation of the relic oscillation energy density in the full five-dimensional limit.

the zero mode, these Kaluza-Klein states dissipate the oscillation energy more efficiently and result in a net decrease in the oscillation energy as a function of time relative to the four-dimensional case. This situation is shown in Fig. 5, where we plot $\rho(t) / \rho_{4 \mathrm{D}}(t)=X\left(\tilde{t}_{0}\right) / X_{4 \mathrm{D}}\left(\tilde{t}_{0}\right)$ as a function of $y \equiv\left(m_{\mathrm{PQ}} R\right)^{-1}$ for three different values of $\tilde{t}_{0}$. Note that in the $y \rightarrow 0, t_{0} \rightarrow 0$ limit, the ratio $\rho(t) / \rho_{4 D}(t)$ decreases as $\sqrt{y}$.

We thus conclude that in such situations, the presence of coupled relic Kaluza-Klein axion oscillations can actually weaken the usual four-dimensional upper bounds on $\hat{f}_{\mathrm{PQ}}$. This implies that it may be possible to consider higher values of $\hat{f}_{\mathrm{PQ}}$ than are usually allowed in four dimensions, thereby further diminishing the axion couplings to matter and providing yet another higher-dimensional method of achieving an "invisible"' axion. We hasten to point out, however, that the size of this effect depends crucially on the value of $y$ as well as on the initial time $\tilde{t}_{0}$ at which the axion potential is established. In general, for a given value of $\tilde{t}_{0}$, we can expect to see a sizable deviation from the four-dimensional asymptotic result only when $\lambda \tilde{t}_{0} \lesssim \mathcal{O}(1)$ for the lightest eigenvalues. This implies $y \tilde{t}_{0} \lesssim \mathcal{O}(1)$. Taking $t_{0}=10^{-5}$ seconds (corresponding to the QCD phase transition) and a reference value
$m_{\mathrm{PQ}}=10^{-4} \mathrm{eV}$, we find $\tilde{t}_{0} \equiv m_{\mathrm{PQ}} t_{0} \approx 10^{6}$. (By contrast, the current cosmological time is given by $\tilde{t} \approx 10^{29}$, corresponding to $t=10^{18}$ seconds.) This implies that in practice, we should not expect to see a sizable decrease in the cosmological relic oscillation energy density unless $y \lessgtr \mathcal{O}\left(10^{-6}\right)$. However, this assumes that a particular value of $m_{\mathrm{PQ}}$, which in turn assumes a particular fixed value of $\hat{f}_{\mathrm{PQ}}$ via Eq. (33). Changing $\hat{f}_{\mathrm{PQ}}$ can therefore change this result substantially. We shall discuss this possibility in Sec. V.

Finally, let us briefly discuss the issue of axion lifetimes as they relate to these cosmological relic oscillations. Ordinarily, relic axion oscillations are important precisely because the usual four-dimensional axion is so long-lived. However, in higher dimensions, we have seen in Sec. III that the heavy Kaluza-Klein axion modes become more and more unstable, and hence cannot be expected to survive over the long cosmological time scales we have been assuming. It is therefore natural to wonder whether this places our conclusions about enhanced energy dissipation rates in jeopardy. In other words, it is not a priori obvious that we are justified in neglecting the excited Kaluza-Klein decay widths in Eq. (77). However, we have seen that relic axion oscillations in higher dimensions are primarily sensitive not to the heaviest axion modes, but to the lightest modes. For sufficiently large radii, these lightest modes have masses which are relatively close to the mass of the axion zero mode, and we have already seen in Fig. 1 that this in turn is bounded from above by the usual four-dimensional axion mass $m_{\mathrm{PQ}}$. Thus, the lightest axion Kaluza-Klein modes continue to be extremely long-lived, and will therefore survive to induce the enhancement of the oscillation energy dissipation rate that we have observed.

\section{SOME NUMBERS, BOUNDS, AND CONSTRAINTS}

Let us now combine our different results from the preceding sections. Our goal will be to determine the extent to which a self-consistent picture of axion energy scales emerges from the previous results.

We begin with the three fundamental equations given in Eqs. (19) and (33). For simplicity, in Eq. (33) we shall take $g^{2}=g_{\text {GUT }}^{2}=1 / 2$, and in Eq. (19) we shall take $\delta=1$. We can therefore combine these three equations in order to express $y$ in terms of the fixed quantities $\Lambda_{\mathrm{QCD}}, M_{\text {string }}$ (the fundamental underlying mass scale in the theory), and the effective axion decay constant $\hat{f}_{\mathrm{PQ}}$ (our measure of "invisibility"). For simplicity we shall also take $f_{\mathrm{PQ}}=M_{\text {string }}$, since we want to have only one fundamental mass scale in the problem. This then yields the result

$$
y \approx \frac{16 \pi^{2}}{\xi} \frac{M_{\mathrm{string}}^{3}}{\hat{f}_{\mathrm{PQ}} \Lambda_{\mathrm{QCD}}^{2}} .
$$

We know that $M_{\text {string }}$ cannot be lower than $\approx \mathcal{O}(1 \mathrm{TeV})$, and $\Lambda_{\mathrm{QCD}}$ is absolutely fixed $(\approx 250 \mathrm{MeV})$. We therefore find that in general, $y$ is bounded from below: 


$$
y \gtrsim y_{\min } \approx \frac{2.5 \times 10^{12} \mathrm{GeV}}{\xi \hat{f}_{\mathrm{PQ}}} .
$$

Thus, assuming $\xi \approx \mathcal{O}(1)$, we see that $\hat{f}_{\mathrm{PQ}} \approx 10^{12} \mathrm{GeV}$ is consistent with having $y \approx \mathcal{O}(1)$. Remarkably, this is precisely the region where we expect to find the axion mass becoming independent of $\hat{f}_{\mathrm{PQ}}$, as shown in Fig. 1.

While this shows the self-consistency of the situation depicted in Fig. 1, a natural question arises as to whether it is possible to tolerate larger values of $M_{\text {string }}$. Indeed, slightly larger values of $M_{\text {string }}$ [e.g., in the $\mathcal{O}(10 \mathrm{TeV})$ range] may be preferred on the basis of detailed comparisons with experimental data. Ordinarily, it might seem to be impossible to increase the value of $M_{\text {string }}$ any further, because we see from Eq. (105) that increasing $M_{\text {string }}$ requires increasing $\hat{f}_{\mathrm{PQ}}$ in order to maintain the $\mathcal{O}(1)$ values of $y$ (as preferred on the basis of Fig. 1), and increasing $\hat{f}_{\mathrm{PQ}}$ generally runs into difficulties with cosmological relic oscillation energy densities overclosing the universe. However, we have seen in Sec. IV that the Kaluza-Klein axion modes may be capable of dissipating this excess energy density more rapidly so as to evade these bounds. The question then arises: to what extent can we increase $\hat{f}_{\mathrm{PQ}}$, thereby making the axion increasingly "invisible," without disturbing the relic energy density bounds?

Note that increasing $\hat{f}_{\mathrm{PQ}}$ has a number of effects. First, as $\hat{f}_{\mathrm{PQ}}$ increases, we find from Eq. (106) that $y_{\text {min }}$ decreases. This means that $y$ can be chosen even smaller. However, we also find from Eq. (33) that $m_{\mathrm{PQ}}$ decreases, which in turn implies that $\tilde{t}_{0} \equiv m_{\mathrm{PQ}} t_{0}$ (the dimensionless time of the QCD phase transition) also decreases. Defining $y_{\text {crit }}$ to be the critical value of $y$ at which we start to observe a significant decrease in the relic oscillation energy density, we have already seen in Sec. IV that $y_{\text {crit }} \approx \tilde{t}_{0}^{-1}$. Thus, as $\tilde{t}_{0}$ decreases, we see that $y_{\text {crit }}$ increases, implying that it becomes easier to compensate for the effect of having increased $\hat{f}_{\mathrm{PQ}}$ in the first place. Indeed, this suggests that there might be an alternative, self-consistent, significantly higher value of $\hat{f}_{\mathrm{PQ}}$ than previously thought.

In order to determine this self-consistent value of $\hat{f}_{\mathrm{PQ}}$, we first note from Eq. (33) that

$$
m_{\mathrm{PQ}} \approx \frac{\left(2.5 \times 10^{-3} \mathrm{GeV}^{2}\right) \xi}{\hat{f}_{\mathrm{PQ}}} .
$$

This in turn implies that

$$
\tilde{t}_{0} \equiv m_{\mathrm{PQ}} t_{0} \approx \frac{\left(3.8 \times 10^{16} \mathrm{GeV}\right) \xi}{\hat{f}_{\mathrm{PQ}}}
$$

where we have taken $t_{0} \approx 10^{-5} \mathrm{sec}$ (corresponding to the QCD phase transition). We thus have

$$
\xi y_{\text {crit }} \approx \frac{\hat{f}_{\mathrm{PQ}}}{3.8 \times 10^{16} \mathrm{GeV}},
$$

which implies that we can obtain $y_{\text {crit }} \approx \mathcal{O}(1)$ simply by taking $\xi \approx \mathcal{O}(1)$ and

$$
\hat{f}_{\mathrm{PQ}} \approx 3.8 \times 10^{16} \mathrm{GeV} \approx M_{\mathrm{GUT}} .
$$

In other words, if we take $\hat{f}_{\mathrm{PQ}} \approx M_{\mathrm{GUT}}$, then we have a selfconsistent solution with $y_{\text {min }} \approx \mathcal{O}(1), y_{\text {crit }} \approx \mathcal{O}(1)$, and $M_{\text {string }} \approx 20 \mathrm{TeV}$. For such values, the axion mass is independent of $\hat{f}_{\mathrm{PQ}}$, and the Kaluza-Klein modes begin to induce a significant reduction in the final relic oscillation energy density which can in principle compensate for the increase in $\hat{f}_{\mathrm{PQ}}$. Remarkably, this analysis suggests that $\hat{f}_{\mathrm{PQ}}$, the effective Peccei-Quinn symmetry-breaking scale, may be related to $M_{\mathrm{GUT}}$, the effective four-dimensional GUT symmetrybreaking scale.

Of course, there is still one constraint that we have not imposed in the above analysis: we have not restricted the size of the radius $R$ of the extra spacetime dimension. In principle, this is not a problem because the standard-model fields are restricted to a D-brane, and thus there are no bounds on the sizes of such transverse extra dimensions that can arise from standard-model processes. However, gravity is generally free to propagate into whatever extra dimensions exist, leading to the additional constraint $R \leqq \mathcal{O}$ (millimeter). It is therefore important to understand how this additional constraint limits the above scenarios. We stress, however, that imposing this additional constraint relies on the assumption that gravity is indeed free to propagate in the extra dimensions. Recent ideas concerning "gravity localization" [20] have shown that this need not always be the case.

If we do assume this to be the case, however, then the above scenarios are significantly restricted. Requiring $R^{-1}$ $\geq 10^{-4} \mathrm{eV}^{\text {implies }}{ }^{7}$ that

$$
m_{\mathrm{PQ}} \gtrsim \frac{10^{-13} \mathrm{GeV}}{y} .
$$

Moreover, using Eq. (107), we find that this implies that

$$
\hat{f}_{\mathrm{PQ}} \lesssim\left(2.5 \times 10^{10} \mathrm{GeV}\right) \xi y .
$$

\footnotetext{
${ }^{7}$ Note that imposing this constraint is actually somewhat subtle, and depends on a choice of which variables to hold fixed. In the analysis in this section, we have been taking $\hat{f}_{\mathrm{PQ}}$ and $y$ as inputs, and treating $M_{\text {string }}, R$, and $m_{\mathrm{PQ}}$ as derived quantities. Thus, with this convention, $\hat{f}_{\mathrm{PQ}}$ is considered to be independent of $R$, while $f_{\mathrm{PQ}}$ (identified with $M_{\text {string }}$ ) is considered to be an $R$-dependent quantity. Note that this procedure exactly mimics the situations $[9,10]$ in which the GUT and Planck scales are lowered by extra spacetime dimensions: it is always the "measured," large, four-dimensional scale that is held fixed, while the reduced higher-dimensional scale is viewed as a function of $R$. We therefore continue this convention in the present case even though neither $\hat{f}_{\mathrm{PQ}}$ nor $f_{\mathrm{PQ}}$ has been experimentally measured.
} 
This therefore sets an upper bound on $\hat{f}_{\mathrm{PQ}}$ as a function of $y$. Combining this upper bound with Eq. (105), we thereby obtain the constraint

$$
\xi y \geq 10\left(\frac{M_{\text {string }}}{1 \mathrm{TeV}}\right)^{3 / 2} .
$$

Thus, the lower limit for $y$ depends crucially on the modeldependent parameter $\xi$ and the value we choose for $M_{\text {string }}$. Since $\xi$ reflects the PQ charges of the ordinary fermions, it is not unreasonable to assume that $\xi$ may be somewhat larger than 1 . Taking $M_{\text {string }} \approx 1 \mathrm{TeV}$ therefore still enables us to have $y \approx \mathcal{O}(1)$. However, despite this fact, we can combine Eq. (109) with Eq. (112) to show that

$$
y_{\text {crit }} \lesssim\left(6.6 \times 10^{-7}\right) y .
$$

Thus the value of $y$ in this case is always significantly larger than the critical value that would be required in order to reduce the relic oscillation energy density below its usual four-dimensional value.

Of course, this does not disturb the self-consistency of this scenario. As a result of Eq. (112), $\hat{f}_{\mathrm{PQ}}$ may still be in the range that satisfies the usual four-dimensional bounds, and we have seen in Sec. IV that the presence of the KaluzaKlein axion states does not increase the final relic oscillation energy density relative to the four-dimensional case. Moreover, these axions continue to be virtually "invisible", against direct detection and/or subsequent interactions as a result of the decoherence effect discussed in Sec. III. Thus this picture continues to be self-consistent, and continues to lead to an invisible axion. Furthermore, although we have restricted our analysis to the case of a single extra dimension, the corresponding constraints in higher dimensions may be significantly weaker. In any case, a much more detailed analysis is necessary in order to make the above numerical bounds more precise, and to determine whether further experimental constraints may be imposed.

There are also other phenomenological constraints that may be imposed, particularly constraints that are insensitive to axion time-evolution. Good examples of this would be axion missing-energy signatures, such as might arise from the decay $K^{+} \rightarrow \pi^{+} a^{\prime}$. In four dimensions, this process has a branching ratio which scales as $\hat{f}_{\mathrm{PQ}}^{-2}$, leading to a bound $\hat{f}_{\mathrm{PQ}} \gtrsim 10^{4} \mathrm{GeV}$. In five dimensions, by contrast, this branching ratio scales as

$$
\mathrm{BR}\left(K^{+} \rightarrow \pi^{+} a^{\prime}\right) \sim \frac{1}{\hat{f}_{\mathrm{PQ}}^{2}} \sum_{n=0}^{R m_{K}} 1 \sim \frac{R m_{K}}{\hat{f}_{\mathrm{PQ}}^{2}} \sim\left(\frac{m_{K}}{M_{\text {string }}}\right) \frac{1}{f_{\mathrm{PQ}}^{2}} .
$$

For $m_{K} \approx 500 \mathrm{MeV}$, this then implies the constraint

$$
f_{\mathrm{PQ}} \gtrsim \sqrt{\frac{m_{K}}{M_{\text {string }}}} 10^{4} \mathrm{GeV} .
$$

Taking $f_{\mathrm{PQ}}=M_{\text {string }}$ then leads to the bound $M_{\text {string }}$ $\gtrsim 370 \mathrm{GeV}$, which is consistent with the idea of lowering the string scale to the TeV-range. Moreover, as we have already mentioned in Sec. III, such a TeV-scale axion scenario is safe with respect to supernova or red-giant bounds for the same reasons that the $\mathrm{TeV}$-scale graviton scenarios are safe [10], namely that supernova and red-giant temperatures are much smaller than the fundamental energy scale of our theory. Of course, we reiterate that a much more detailed analysis of these and other processes is necessary in order to sharpen these bounds and constraints.

Finally, it may also happen that an axion process in higher dimensions exactly reproduces the four-dimensional result. As an example of this, let us consider an $a^{\prime}$-exchange process at zero momentum transfer (e.g., $F \widetilde{F} \rightarrow a^{\prime} \rightarrow F \widetilde{F}$ ). The amplitude for such a process is given by

$$
A=\frac{1}{f_{\mathrm{PQ}}^{2}}\left\langle a^{\prime} a^{\prime}\right\rangle \sim \frac{1}{\hat{f}_{\mathrm{PQ}}^{2}} \sum_{m, n=0}^{\infty} r_{m} r_{n}\left\langle a_{m} a_{n}\right\rangle
$$

where $\langle B A\rangle$ denotes the propagator from state $A$ to state $B$. Passing to the mass-eigenstate basis $\hat{a}_{\lambda}$ via Eq. (39) and using the zero-momentum propagator $\left\langle\hat{a}_{\lambda^{\prime}}, \hat{a}_{\lambda}\right\rangle=\delta_{\lambda^{\prime} \lambda} / \lambda^{2}$, we find that this amplitude then takes the form

$$
A=\frac{1}{\hat{f}_{\mathrm{PQ}}^{2} m_{\mathrm{PQ}}^{2}} \sum_{\lambda} \frac{1}{\widetilde{\lambda}^{2}} \sum_{m, n=0}^{\infty} r_{m} r_{n} U_{\lambda m} U_{\lambda n}=\frac{1}{\hat{f}_{\mathrm{PQ}}^{2} m_{\mathrm{PQ}}^{2}} .
$$

Note that we have used Eq. (44) followed by Eq. (43) in the final equality. However, we see that the final result is nothing but the amplitude that we would have obtained in four dimensions for an axion that couples with the usual fourdimensional coupling $\hat{f}_{\mathrm{PQ}}$ and has the usual four-dimensional mass $m_{\mathrm{PQ}}$. Thus, in this case, we obtain no new bounds coming from such $a^{\prime}$-mediated processes. This stands in stark contrast to the analogous case of graviton-mediated processes, from which one can generally derive stringent bounds on the radii of the extra spacetime dimensions. Of course, we stress that this result holds only for zero momentum transfer, and is likely to be different when sizable momenta are carried by the intermediate axion state.

\section{THE STANDARD-MODEL DILATON}

The above considerations about placing a standard-model singlet field in the bulk are actually quite general, and transcend the specific example of the QCD axion. To illustrate this point, let us briefly consider the case of another conjectured particle, the standard-model dilaton. This particle is introduced into the standard model in order to restore the classical scale invariance broken by mass terms. The scaleinvariant extension of the scalar sector of standard model is given by [22]

$$
\mathcal{L}=\frac{1}{2}\left(\partial_{\mu} D\right)^{2}+\left(D_{\mu} \phi\right)\left(D^{\mu} \phi\right)^{\dagger}-V_{0}(\phi, \sigma)
$$

where $\phi$ is the Higgs field and where the standard-model dilaton field $D$, like the axion field $a$, is written in terms of a decay constant $f_{D}$ via a relation of the form

$$
D=f_{D} \exp \left(\sigma / f_{D}\right) .
$$


By suitably choosing the parameters in the tree-level Higgs potential $V_{0}(\phi, \sigma)$, we can arrange $\langle\sigma\rangle=0$. Consequently $\langle D\rangle=f_{D}$ represents the mass scale at which dilation invariance is spontaneously broken. After quantum corrections are included, the scalar potential can be written in the form [23]

$$
V=\frac{D^{4}}{f_{D}^{4}} \mathcal{V} \text { where } \mathcal{V} \equiv\left[V_{0}(\phi, 0)+V^{(1)}(\phi, 0)-\Delta(\phi, 0) \ln \frac{D}{f_{D}}\right] \text {. }
$$

Here $V^{(1)}(\phi, 0)$ is the one-loop contribution to the effective potential and $\Delta(\phi, 0)$ is the divergence of the dilation current. The presence of $\Delta(\phi, 0)$ in this expression breaks the scale invariance, and gives rise to a dilaton mass

$$
m_{D}^{2}=-\frac{4\langle\Delta\rangle}{f_{D}^{2}}
$$

where $\langle\mathcal{V}\rangle=\Delta / 4$ at the minimum of the potential (121). Note that in the standard model, the heavy top-quark mass leads to $\langle\Delta\rangle>0$. Thus, in order to change the sign of $\langle\Delta\rangle$, one requires additional heavy Higgs-boson contributions for the stability of the dilaton potential.

Let us now consider what happens when the dilaton field propagates in a five-dimensional bulk and therefore has a Kaluza-Klein decomposition of the form

$$
D\left(x^{\mu}, y\right)=\sum_{n=0}^{\infty} D_{n}\left(x^{\mu}\right) \cos \left(\frac{n y}{R}\right) .
$$

The five-dimensional action for the dilaton then takes the form

$$
\mathcal{S}=\int d^{4} x d y M_{s}\left[\frac{1}{2}\left(\partial_{M} D\right)^{2}-V(x) \delta(y)\right]
$$

where $V$ is given in Eq. (121). Substituting Eq. (123) into Eq. (124) and integrating over the fifth dimension then gives rise to the effective four-dimensional Lagrangian

$$
\begin{aligned}
\mathcal{L}_{\text {eff }}= & \frac{1}{2} \sum_{n=0}^{\infty}\left(\partial_{\mu} D_{n}\right)^{2}-\frac{1}{2} \sum_{n=1}^{\infty} \frac{n^{2}}{R^{2}} D_{n}^{2}-\frac{1}{\hat{f}_{D}^{4}}\left(\sum_{n=0}^{\infty} r_{n} D_{n}\right)^{4} \\
& \times\left[V_{0}(\phi, 0)+V^{(1)}(\phi, 0)-\Delta(\phi, 0) \ln \left(\frac{1}{\hat{f}_{D}} \sum_{n=0}^{\infty} r_{n} D_{n}\right)\right]
\end{aligned}
$$

where $\hat{f}_{D} \equiv \sqrt{2 \pi R M_{\text {string }}} f_{D}$ and where we have canonically normalized the dilaton kinetic terms. The minimum of the effective dilaton potential therefore occurs at

$$
\begin{aligned}
& \left\langle D_{0}\right\rangle=\hat{f}_{D} \\
& \left\langle D_{n}\right\rangle=0 \text { for all } n \geqslant 1 .
\end{aligned}
$$

We can derive the dilaton mass matrix by considering the local curvature of the effective dilaton potential near its minimum. This is completely analogous to the axion case (30), and gives rise to

$$
\left(\mathcal{M}_{D}\right)_{n n^{\prime}}^{2} \equiv \frac{n^{2}}{R^{2}} \delta_{n n^{\prime}}-\frac{4 \Delta}{\hat{f}_{D}^{2}} r_{n} r_{n^{\prime}} .
$$

Remarkably, this mass matrix has exactly the same structure as in the axion case (32), and consequently the physical implications will be identical to those for the axion. Of course, this result is expected since the dilaton and axion are both Nambu-Goldstone bosons of a spontaneously broken symmetry, and consequently have similar couplings to the anomalous divergences of their respective currents.

\section{CONCLUSIONS}

In this paper, we have studied some of the novel effects that arise when the QCD axion is free to propagate in the bulk of large extra spacetime dimensions. First, we found that under certain circumstances, the mass of the axion can become independent of the energy scale associated with the breaking of the Peccei-Quinn symmetry. This feature does not arise in four dimensions. Because this energy scale determines the couplings between the axion and ordinary matter, this suggests that in higher dimensions, one has the freedom to adjust the strength of the axion couplings without disturbing the axion mass. This can therefore provide a new mechanism for achieving an invisible axion.

Second, we pointed out that in such higher-dimensional scenarios, the axion will typically experience laboratory axion oscillations which are completely analogous to neutrino oscillations. This is therefore a new and unexpected phenomenological feature for axions that does not exist in the usual four-dimensional case. Moreover, we found that these laboratory oscillations can cause axions to "decohere" extremely rapidly. This is therefore a second higherdimensional phenomenon that may contribute to an invisible axion. Moreover, this phenomenon arises for all non-zero radii.

Third, we discussed the role that excited Kaluza-Klein axion states may play in axion-mediated processes and axion decays. This enabled us to propose several direct experimental tests of the proposed higher-dimensional nature of the axion.

Finally, we found that under certain circumstances, the presence of these Kaluza-Klein axion modes can significantly accelerate the dissipation of the energy associated with cosmological relic axion oscillations. Moreover, even when these circumstances are not met, we found that the Kaluza-Klein states do not induce a violation of the usual four-dimensional bounds. This demonstrates that such higher-dimensional axion scenarios are no less viable than their four-dimensional counterparts, and indeed may even be preferred on the basis of their remarkable "invisibility" decoherence properties.

Of course, there are many aspects of higher-dimensional axion phenomenology which we have not examined in this paper. These include the role that axions play in stellar evolution, the thermal production of axions, axionic string decay, and isocurvature axion fluctuations. While some of these topics have been discussed in Ref. [13], it will be in- 
teresting to further explore the role that extra spacetime dimensions can play in these areas.

Although we have focused primarily on the case of QCD axions, we stress that much of our analysis is completely general and may apply for other bulk fields as well. This was explicitly illustrated in Sec. VI, where we considered the case of the standard-model dilaton. Similarly, we expect that our analysis will also apply to other bulk fields such as Kaluza-Klein gravitons, string-theoretic dilatons, and other bulk moduli. Indeed, the twin properties of laboratory oscillations and decoherence leading to "invisibility" are likely to play an important role in experimental searches for such particles, and likewise an analysis of the effects of their ex- cited Kaluza-Klein modes on cosmological evolution is likely to parallel the analysis in Sec. IV. Moreover, both of these effects are likely to play an important role in the allimportant questions of dilaton and radion stabilization. We therefore leave these issues for future investigation.

\section{ACKNOWLEDGMENTS}

We wish to thank K. Orginos, I. Sarcevic, and especially J. Mourad for useful discussions. K.R.D. and T.G. also wish to acknowledge the hospitality of the Aspen Center for Physics where part of this work was done.
[1] S. Weinberg, Phys. Rev. D 11, 3583 (1975).

[2] G. 't Hooft, Phys. Rev. Lett. 37, 8 (1976); Phys. Rev. D 14, 3432 (1976).

[3] For a review, see R.D. Peccei, in CP Violation, edited by C. Jarlskog (World Scientific, Singapore, 1989), pp. 503-551.

[4] R.D. Peccei and H.R. Quinn, Phys. Rev. Lett. 38, 1440 (1977); Phys. Rev. D 16, 1791 (1977).

[5] S. Weinberg, Phys. Rev. Lett. 40, 223 (1978).

[6] F. Wilczek, Phys. Rev. Lett. 40, 279 (1978).

[7] For a review, see J. Kim, Phys. Rep. 150, 1 (1987).

[8] I. Antoniadis, Phys. Lett. B 246, 377 (1990); I. Antoniadis, K. Benakli, and M. Quirós, ibid. 331, 313 (1994).

[9] K.R. Dienes, E. Dudas, and T. Gherghetta, Phys. Lett. B 436, 55 (1998); Nucl. Phys. B537, 47 (1999); hep-ph/9807522.

[10] N. Arkani-Hamed, S. Dimopoulos, and G. Dvali, Phys. Lett. B 429, 263 (1998); Phys. Rev. D 59, 086004 (1999); I. Antoniadis et al., Phys. Lett. B 436, 257 (1998).

[11] E. Witten, Nucl. Phys. B471, 135 (1996); J.D. Lykken, Phys. Rev. D 54, 3693 (1996); G. Shiu and S.-H.H. Tye, ibid. 58, 106007 (1998).

[12] K. Benakli, Phys. Rev. D 60, 104002 (1999); C.P. Burgess,
L.E. Ibáñez, and F. Quevedo, Phys. Lett. B 447, 257 (1999).

[13] S. Chang, S. Tazawa, and M. Yamaguchi, Phys. Rev. D 61, 084005 (2000); hep-ph/9909240.

[14] K.R. Dienes, E. Dudas, and T. Gherghetta, Nucl. Phys. B557, 25 (1999).

[15] N. Arkani-Hamed et al., hep-ph/9811448.

[16] G. Shiu, R. Shrock, and S.-H.H. Tye, Phys. Lett. B 458, 274 (1999).

[17] M. Bando et al., Phys. Rev. Lett. 83, 3601 (1999); J. Hisano and N. Okada, Phys. Rev. D 61, 106003 (2000).

[18] E. Dudas and J. Mourad, Nucl. Phys. B575, 3 (2000).

[19] D. Gross, R. Pisarski, and L. Yaffe, Rev. Mod. Phys. 53, 43 (1981); M.S. Turner, Phys. Rev. D 33, 889 (1986).

[20] L. Randall and R. Sundrum, Phys. Rev. Lett. 83, 4690 (1999).

[21] L. Di Lella et al., hep-ph/0006327.

[22] For an introductory discussion, see S. Coleman, "Dilatations," in Aspects of Symmetry: Selected Erice Lectures (Cambridge University Press, Cambridge, England, 1985), pp. 6798.

[23] R.D. Peccei, J. Sola, and C. Wetterich, Phys. Lett. B 195, 183 (1987); W. Buchmüller and N. Dragon, ibid. 195, 417 (1987). 